\title{
Performance Analysis and Optimal Tuning of IETF LPWAN SCHC ACK-on-Error Mode
}

\author{
Sergio Aguilar, Patrick Maillé, Laurent Toutain, Carles Gomez, Rafael Vidal, Nicolas Montavont, Georgios \\ Z. Papadopoulos
}

\begin{abstract}
The Internet Engineering Task Force (IETF) Low Power Wide Area Network (LPWAN) Working Group has developed the Static Context Header Compression (SCHC) framework to enable IPv6 over LPWAN. In order to support 1280-byte packets, as required for IPv6, SCHC includes a fragmentation functionality, since relevant LPWAN technologies offer very short data unit sizes and do not provide native fragmentation mechanisms. SCHC offers 3 fragmentation modes: No-ACK, ACK-Always, and ACK-on-Error, the latter being especially promising due to its reliability and high efficiency. In this article, we develop a mathematical model to compute the most critical performance parameters for the SCHC ACK-on-Error mode, namely, the acknowledgment traffic incurred

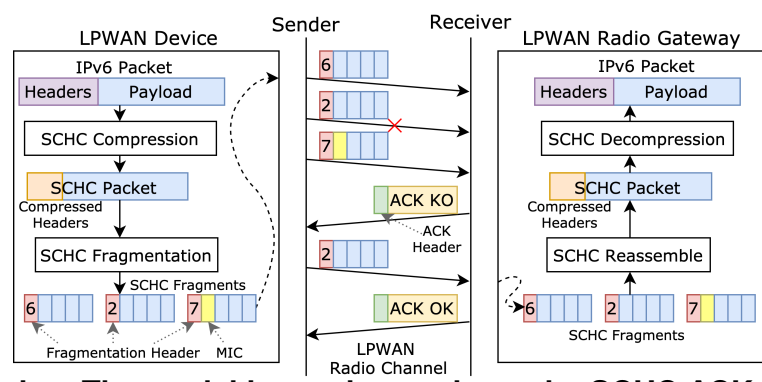
by a fragment receiver for the successful delivery of a fragmented packet. The model is used to evaluate the SCHC ACKon-Error mode performance, as well as to optimally tune its main parameters when used over LoRaWAN and Sigfox, for different packet sizes. Additionally, we illustrate how our derived optimal settings allow to reduce the acknowledgment traffic in a number of scenarios.
\end{abstract}

Index Terms - LPWAN, SCHC, ACK-on-Error, LoRaWAN, Sigfox, loT, LoRa, IETF, mathematical model, fragmentation

\section{INTRODUCTION}

$\mathbf{L}$ OW Power Wide Area Networks (LPWANs) refer to network technologies designed for the Internet of Things (IoT) that are characterized by a long-range and low-energy operation [1-6]. LPWAN technologies are based on star topology deployments, where a potentially high number of Internet of Things (IoT) devices are directly connected to a radio gateway.

In some quintessential LPWAN technologies, such as LoRaWAN and Sigfox, the layer 2 Maximum Transmission Unit (L2 MTU) ranges from tens to hundreds of bytes [1]. To carry IPv6 packets over LPWAN despite those limitations, the Internet Engineering Task Force (IETF) has defined a new adaptation layer called Static Context Header Compression (SCHC) [7], [8], which along with a header compression functionality, provides fragmentation mechanisms to transport an IPv6 packet over several LPWAN frames. SCHC defines 3 Fragmentation/Reassembly (F/R) modes called No-ACK,

This work was supported in part by the Spanish Government through project TEC2016-79988-P, AEI/FEDER, EU and by Secretaria d'Universitats i Recerca del Departament d'Empresa i Coneixement de la Generalitat de Catalunya 2017 SGR 376.

S.Aguilar, C. Gomez and R. Vidal are with the Department of Network Engineering, Universitat Politècnica de Catalunya, 08860 Castelldefels, Barcelona, Spain (e-mail: sergio.aguilar.romero@upc.edu, \{carlesgo, rafael.vidal\}@entel.upc.edu)

P. Maillé, L. Toutain, N. Montavont and G. Z. Papadopoulos are with the Department of Network Systems, Cyber Security and Digital Law (SCRD), IMT-Atlantique, IRISA, 35576 Campus of Rennes, France (e-mail: \{patrick.maille, laurent.toutain, nicolas.montavont, georgios.papadopoulos\}@imt-atlantique.fr)
ACK-Always and ACK-on-Error. This paper focuses on the ACK-on-Error mode, which is promising due to its reliability and high efficiency by minimizing the number of acknowledgments (ACKs) compared to ACK-Always. In this mode, ACKs are sent by the fragment receiver only when the latter detects fragment losses. Then, the fragment sender selectively retransmits any lost fragments reported in the ACKs. To maintain consistency, a final ACK is also unconditionally sent at the end of the fragmented packet transmission.

A key constraint for LPWAN is the amount of downlink traffic, i.e., from the gateway to the IoT device. Indeed, in the spectrum band used by unlicensed LPWAN technologies in Europe (e.g., the $868 \mathrm{MHz}$ band), each device must respect a duty-cycle limit, that can be especially binding for gateways, which may manage many flows. Even if the downlink traffic is not limited by the duty-cycle constraint, one may still want to minimize it for economic reasons: the amount of downlink traffic is now used by some operators as a basis for charging IoT users in some plans ${ }^{1}$ or is limited [4]. Noting that IoT flows are mostly uplink flows (e.g., from the IoT device, say a sensor, sending its data readings), the focus in this paper is on the ACK traffic incurred by the SCHC F/R processthe main expected type of downlink traffic-which needs to be minimized in order to reduce costs and/or respect gateway duty-cycle constraints [9].

More specifically, in this paper we propose a mathematical

\footnotetext{
${ }^{1}$ See, e.g., https://iotmarket.orange.com/connectivity. html (accessed on 30/06/2019)
} 
model to compute the volume of ACK traffic (relative to the IoT device data volume) based on the quality of the radio link and the SCHC F/R parameters. We then use the model to optimize those parameters in order to minimize the ACK traffic. For a direct practical use of our results, we provide the optimal parameter values for the specifics of LoRaWAN [10] and Sigfox [11] technologies.

The remainder of this paper is organized as follows. In Section II we first present the existing works related to LPWAN fragmentation and its performance. We then detail the SCHC framework in Section III, and the SCHC F/R modes in Section IV, especially focusing on the ACK-on-Error mode. The mathematical model used to analyze the ACK-on-Error mode is developed in Section V, and it is used in Section VI to evaluate the performance metrics, mostly regarding the amount of ACK messages. Section VII explains how our results can be applied to state-of-the-art LPWAN technologies such as LoRaWAN and Sigfox. Finally, we provide some conclusions in Section VIII.

\section{Related Work}

Recent attention on LPWAN technologies has partly focused on the evaluation of the physical and link layers [2], [4], [5], [9]. In [9] the authors analyze LoRaWAN and explore its limitations. The results show that the application and network design must minimize the number of acknowledged frames to avoid capacity drain, because the LPWAN gateway must enforce a time-off following the transmission to comply with duty-cycle regulations. Other works provide a mathematical model that characterizes LoRaWAN and Sigfox end-device energy consumption, lifetime and energy involved in data delivery [2], [4]. In [5] a performance evaluation of Sigfox scalability is presented. Together, these studies provide important insights into the physical and link layers of LPWAN technologies, but do not consider the compatibility with IPv6, nor fragmentation mechanisms.

Several studies compare different LPWAN technologies [3], [6], [12]. While Mroue et al. [3] perform an evaluation using the packet error rate for Sigfox, LoRa and NB-IoT, a comprehensive and comparative study for a number of performance metrics is presented in [12]. The study in [6] evaluates, by simulation, the influence of the number of devices, on the packer error rate, collisions and spectrum utilization for Sigfox and LoRa. None of these studies address the problem of transmitting a fragmented IPv6 packet over LPWAN.

Regarding the upper layer functionalities in LPWAN, the study in [13] evaluates the effect of fragmentation, and its efficiency in terms of energy consumption, throughput, goodput and average delay in dense networks. Suciu et al [13] showed that fragmentation increases reliability, especially when sending several fragments instead of only one of the MTU size. Other works focus on IPv6 over LPWAN by means of using SCHC [8], [14-18]. Some of these propose enhancements to SCHC Header compression, but do not consider SCHC F/R [16], [17]. On the other hand, Moons et al. [14] compared SCHC and 6LoWPAN compression and fragmentation functionalities. Their results show that SCHC has a smaller footprint, uses less memory and the header overhead is twenty times smaller when compared with 6LoWPAN. Ayoub et al. [15] present an implementation of SCHC using the ns-3 network simulator and also compare SCHC with 6LoWPAN, finding the same performance advantage for SCHC in terms of header overhead. The authors in [8] provided an overview of SCHC and a simple evaluation of the different F/R modes, but it is a superficial study due to its tutorial purpose. In [18], the authors compared the different SCHC F/R modes in terms of total channel occupancy, goodput and total delay at the SCHC layer in an ideal communication channel. The authors showed that, when comparing the reliable SCHC F/R modes, namely, ACK-Always and ACK-on-Error, the latter provided better goodput.

To the best of our knowledge, no previous work provides a mathematical model to estimate the ACK volume and its relation with key configuration parameters of SCHC F/R ACKon-Error mode, nor contribute with configuration guidance based on radio link quality and packet size. We think that this mode is worth analyzing, because it provides reliable communication while minimizing the number of ACKs when compared to ACK-Always.

\section{TECHNICAL BACKGROUND: SCHC FRAGMENTATION AND REASSEMBLY}

This section provides an overview of SCHC F/R. We first introduce the SCHC adaptation layer, then focus on the main SCHC F/R components and tools.

\section{A. SCHC Adaptation Layer Overview}

Flagship LPWAN technologies, such as LoRaWAN and Sigfox, are characterized by a reduced L2 MTU [1]. Furthermore, these technologies do not provide a native fragmentation mechanism for transferring larger packets. The SCHC framework provides header compression and $\mathrm{F} / \mathrm{R}$ functionalities specifically designed for LPWAN [7]. SCHC defines a set of Rules, each identified with a RuleID, which determine how to perform the compression and fragmentation and allow the sender and receiver to determine the operation mode and configuration parameters.

SCHC is composed of two sublayers, namely the Compression and the Fragmentation sublayers. Fig. 1 shows those sublayers between the IPv6 layer and the LPWAN technology layer. When an IPv6 packet needs to be sent, compression is

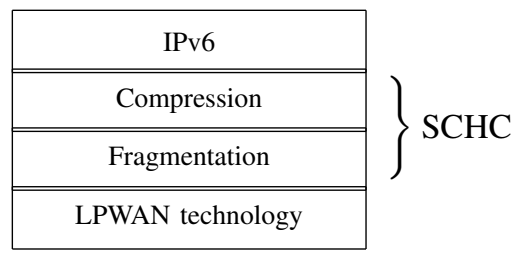

Fig. 1. Protocol stack illustrates the location of the SCHC sublayers between the IPv6 layer and the underlying LPWAN technology [7].

performed. The compressed IPv6 is called a SCHC Packet. If the SCHC Packet size is greater than the L2 MTU, SCHC fragmentation is performed at the sender. At the receiver, 
the SCHC Packet is reassembled and the IPv6 packet is decompressed.

In SCHC F/R, a SCHC Packet is fragmented into units called tiles. One or more tiles are carried by one SCHC Fragment, which is sent in an LPWAN frame. In some SCHC $\mathrm{F} / \mathrm{R}$ modes, a determined number of tiles are grouped into a window, and the receiver generates SCHC ACKs to tell the sender which tiles of that window have been received or not. Missing fragments or tiles are retransmitted. Tiles and windows are numbered in a way that each tile can be identified for further retransmissions (see Fig. 2).

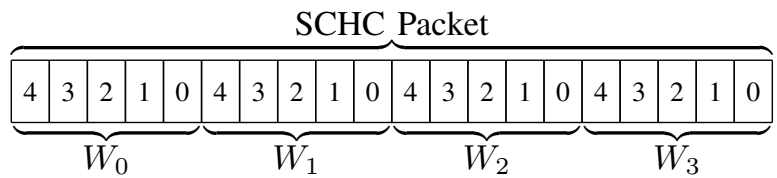

Fig. 2. Example of a SCHC Packet fragmented into 20 tiles, with 5 tiles per window. The tile index is indicated for each tile.

\section{B. SCHC F/R Messages and Headers}

The SCHC framework defines different messages that are used to carry out the SCHC F/R process between the sender and the receiver. The main messages are the SCHC Fragment and the SCHC ACK. Each message has a SCHC F/R Header with the following fields:

- Rule ID: this field identifies whether a SCHC message is a SCHC Fragment. In a SCHC Fragment, it indicates which F/R mode and settings are used.

- Datagram Tag (DTag): this field is used to identify-along with the Rule ID-a SCHC Packet. The length of the DTag field is $T$ in bits.

- $W$ : this number identifies the window a fragment belongs to and has a length of $M$ bits. $W$ is only present in SCHC F/R modes that use windows.

- Fragment Compressed Number (FCN): this $N$-bit field is used to identify the progress of the sequence of tile(s) being transmitted in a SCHC Fragment message.

- Reassembly Check Sequence (RCS): this field, of $U$ bytes, is used to check the integrity of a reassembled SCHC Packet. It protects the complete SCHC Packet.

- Integrity Check (C): this one-bit field equals 1 if the integrity check of the reassembled SCHC Packet succeeded, and 0 otherwise.

A SCHC Fragment carries a part of a SCHC Packet from the sender to the receiver. The FCN field of a SCHC Fragment has all bits set to 1 (it is then called an All-1 SCHC Fragment), to indicate it is the last fragment for the current SCHC Packet; that fragment carries the RCS for this SCHC Packet. Fig. 3 shows a regular and an All-1 SCHC Fragment. $L_{\mathrm{SH}}$ is the length of the SCHC Fragment Header in bytes. Padding bits are added at the end of the SCHC Fragment if needed by the LPWAN technology. A SCHC ACK is sent by the receiver to the sender to acknowledge the complete or partial reception of the fragmented SCHC Packet. In the latter case, a SCHC ACK reports whether the tiles of a given window have been
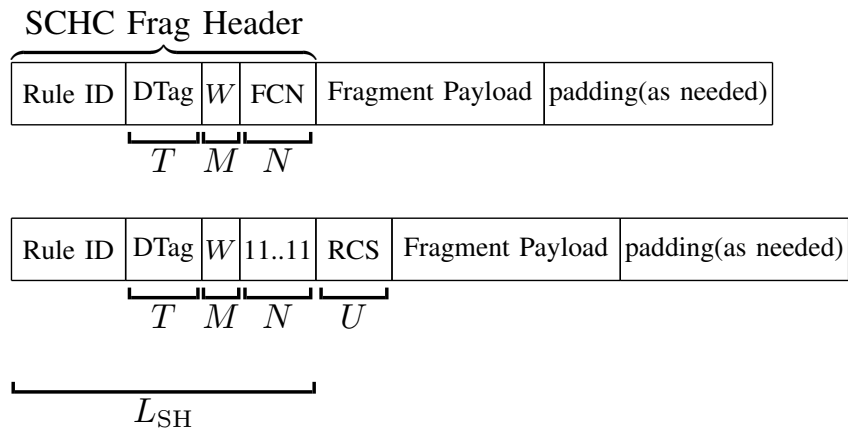

Fig. 3. Illustration of a regular SCHC Fragment, and an All-1 SCHC Fragment with the RCS field. The length of each header field is indicated below each field.

SCHC ACK Header

\begin{tabular}{|c|c|c|c|c|c|c|}
\hline Rule ID & DTag $W$ & $\mathrm{C}=1$ & \multicolumn{2}{|c|}{ padding (as needed) } & (success & \\
\hline Rule ID & DTag $W$ & $\mathrm{C}=0$ & Bitmap & padding (as & leeded) & (failure) \\
\hline
\end{tabular}

Fig. 4. Illustration of a SCHC ACK message. The top SCHC ACK message notifies successful reassembly of a SCHC Packet by carrying a $C=1$. The bottom one indicates a failed SCHC Packet reassembly $(C=0)$ and carries a bitmap. The length of each header field is indicated below each field.

received or not, in the form of a bitmap (see section III-E). Fig. 4 shows the SCHC ACK format. A SCHC ACK carries the $C$ field.

\section{Tiles}

As previously explained (see Fig. 2), a SCHC Packet is fragmented into units called tiles, which have a size of $t$ bytes. In ACK-on-Error mode, each tile must be of the same size, except for the last one, which can be smaller. In the No-ACK and ACK-Always modes, tiles can be of different sizes. If the payload field is present in a SCHC Fragment, it must carry at least one tile. In ACK-on-Error mode, each tile of a SCHC Packet is uniquely identified by the window and tile numbers. The FCN of the SCHC Fragment, together with the window index $\left(W_{i}\right)$, identifies the first tile carried by the SCHC Fragment.

\section{Windows}

A group of $w$ successive tiles is called a window. Each window in a fragmented SCHC Packet transmission, except the last one, must have the same number of tiles. Windows are numbered from 0 upwards. The window field $(W)$ has a size of $M$ bits and the window size $(w)$ has to be less than $2^{N}$ (in each window, the tiles are numbered from $w-1$ downwards). Fig. 2 shows the fragmentation of a SCHC Packet in 4 windows, with 5 tiles per window. 


\section{E. Bitmap}

A bitmap is a sequence of bits where each bit indicates the received status of a tile within a specific window. The bitmap has a size of $w$. The rightmost and leftmost bits will correspond to tile numbers 0 and $w-1$, respectively. The receiver will set a 1 in the bitmap when the corresponding tile is received successfully and a 0 when the tile was not received, as exemplified in Fig. 5. The $C$ field is set to 0, indicating the packet reassembly was not successful, since the SCHC Packet was not received completely.

\begin{tabular}{l|c|c|c|c|c|c|c|c|c|c|c|}
\hline RCHC ACK Header & \multicolumn{1}{c|}{ Bitmap } \\
\hline Rule ID & DTag & $W$ & $\mathrm{C}=0$ & 0 & 1 & 1 & 1 & 1 & 1 & 1 & padding(as needed) \\
tile number $=$ & 6 & 5 & 4 & 3 & 2 & 1 & 0
\end{tabular}

Fig. 5. Example of a SCHC ACK, where a window of 7 tiles $(\boldsymbol{w}=7)$ was sent and tile FCN \# 6 was not received successfully.

\section{SCHC Fragmentation and Reassembly Modes}

SCHC offers 3 SCHC F/R modes to perform the SCHC F/R process: No-ACK, ACK-Always and ACK-on-Error. If a reliable communication is required, ACK-Always and ACKon-Error use ACKs to support potential retransmission upon failure. This section provides a brief description of No-ACK and ACK-Always modes, and a more detailed explanation of the ACK-on-Error mode.

\section{A. The No-ACK mode}

The No-ACK mode provides a mechanism for in-sequence delivery of SCHC Fragments between the sender and the receiver. This mode does not provide reliability when errors are present, since there is no feedback from the receiver and the sender cannot perform SCHC Fragment retransmissions. Variable L2 MTU size is supported. Tiles can be of different sizes, while windows are not used.

\section{B. ACK-Always mode}

The ACK-Always mode is a window-based mechanism for in-sequence delivery of SCHC Fragments that supports reliability. At the end of the transmission of each window, a SCHC ACK is sent by the receiver to the sender to report on the tiles received for the current window. The sender only begins the transmission of the next window, once the receiver confirms the correct reception of all tiles of the current window. Variable L2 MTU size is not supported. Tiles can have different sizes.

\section{ACK-on-Error mode}

The ACK-on-Error mode is a window-based mechanism that supports reliable and out-of-order delivery of SCHC Fragments and variable L2 MTU. This SCHC F/R mode reduces the number of SCHC ACKs, when compared to ACKAlways, since in all windows except for the last one carrying a SCHC Packet, SCHC ACKs are only sent when at least

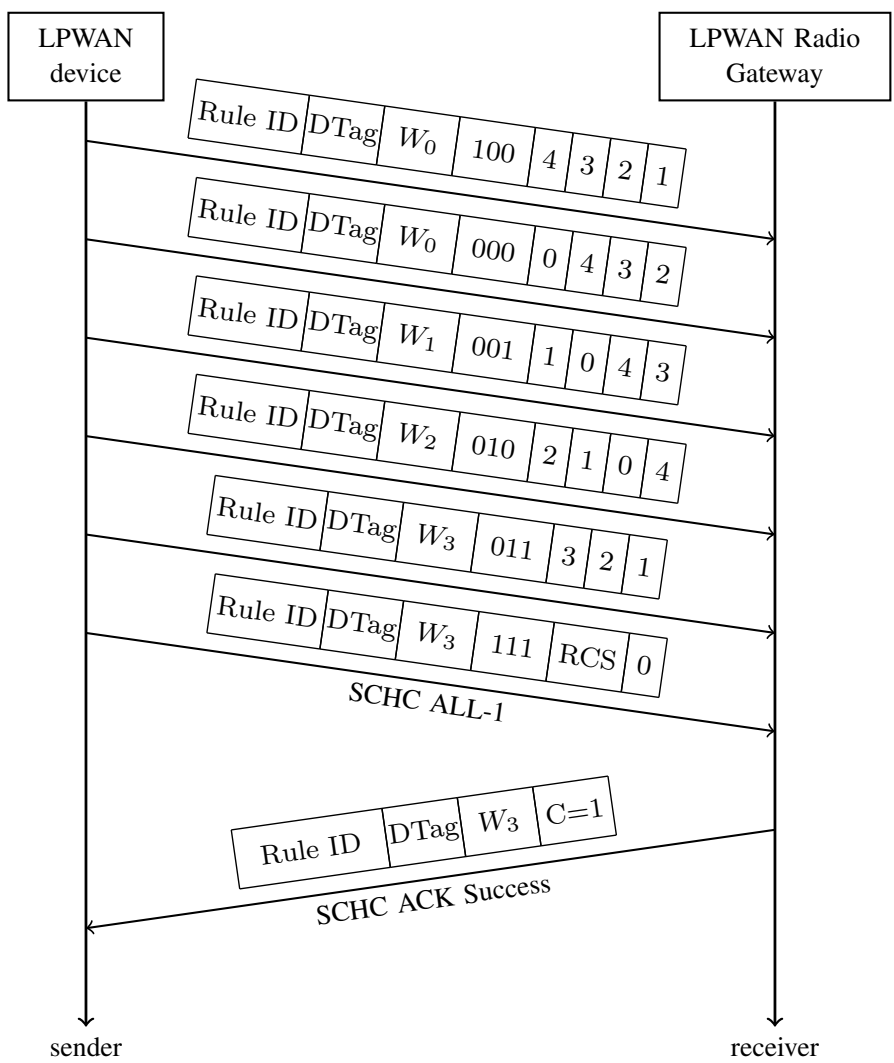

Fig. 6. Example of a transmission of the SCHC Packet shown in Fig. 2 with no errors. The transmission ends with a SCHC ACK that indicates successful SCHC Packet reassembly.

one tile is lost. For the last window, in order to ensure that the sender can expect to receive feedback on the fragmented SCHC Packet transmission, a SCHC ACK is unconditionally sent.

All tiles must be of the same size, except for the last one, which can be smaller. One or more tiles can be carried in a SCHC Fragment and they can be from multiple windows.

In a SCHC Fragment, the window number $(W)$ needs to be set to identify each window number unambiguously during the transmission of a SCHC Packet. The sender can retransmit the SCHC Fragments for any lost tiles, from previous windows. This allows the sender and the receiver to work in a loosely coupled manner.

Transmission of a fragmented SCHC Packet may end in three ways: first when the integrity check for the SCHC Packet shows a correct reassembly at the receiver, second when too many retransmission attempts were made, finally when an inactivity timer at the receiver indicates that the transmission has been inactive for too long. Fig. 6 shows the transmission of the fragmented SCHC Packet of Fig. 2 with no errors, where the SCHC Fragment size allows 4 tiles per fragment.

If the receiver receives the All-1 SCHC Fragment, it performs the integrity check for the SCHC Packet. This is carried out by comparing the RCS calculated with the RCS received in the All-1 SCHC ACK Fragment. With the result of the Integrity Check, the $C$ field is populated with 1 or 0 , indicating success or failure of the SCHC Packet reassembly, respectively. In case some tiles of a window or a complete 
window were lost, the receiver prepares the bitmap for the lowest-numbered window that was not entirely received.

The sender has to listen for a SCHC ACK from the receiver after sending the All-1 SCHC Fragment. Moreover, a technology-oriented profile specification can establish other times when the sender may need to listen for a SCHC ACK, for example after sending a complete window of tiles. The sender can terminate the transmission of a SCHC Packet when receiving a SCHC ACK with $C=1$. If the SCHC ACK carries $C=0$, the sender must resend the SCHC Fragments corresponding to the missing tiles indicated in the bitmap. As an example, Fig. 7 shows the transmission of the SCHC Packet presented in Fig. 2 with an error in the 4th SCHC Fragment. Even though the SCHC Fragment carries tiles from 2 windows, the SCHC ACK indicates the window number of the lower-numbered window and the sender is able to identify which SCHC Fragment has to be retransmitted. After the retransmission of the missing SCHC Fragment, the receiver computes the RCS, performs the integrity check and sends a SCHC ACK reporting successful SCHC Packet reassembly.

\section{Mathematical Model And Analysis}

This section provides the mathematical model used to calculate the expected number of SCHC ACKs, hereafter called ACKs, required to successfully transfer a fragmented SCHC Packet in ACK-on-Error mode, in presence of losses. On this basis, the section also introduces a number of related crucial performance metrics. Sections V-A to V-D present how to compute the performance metrics, namely: ACK message overhead, ACK bit overhead, ACK bit overhead with L2 Headers and percentage of used bits per fragment, respectively. In addition, Section V-E presents useful parameters such as the maximum window size and the maximum bitmap sizes.

For the analysis we consider an infinite maximum number of fragment retries, all tiles of the same size and no padding in the bitmap. We do not count the unconditional SCHC ACK (see Fig. 4) generated by the receiver to notify that all tiles of a SCHC Packet have been received successfully because it does not provide more information to the analysis.

\section{A. ACK Message Overhead}

The ACK message overhead $\left(E_{k}\right)$ is defined as the expected number of ACKs required to successfully transfer a given window. $E_{k}$ will depend on the average number of SCHC Fragments necessary to transmit all the tiles of a window and on the probability that each SCHC Fragment is successfully received.

If we assume that all bits must be received without errors for the transmission to be successful, the probability of success $\left(P_{\mathrm{S}}\right)$ for a SCHC Fragment can be related to the Bit Error Rate (BER) through the relation

$$
P_{\mathrm{s}}=(1-B E R)^{8 F}
$$

where $F$ is the fragment size in bytes of the L2 MTU of the underlying LPWAN technology. (Note that we implicitly assume a uniform BER that refers to the residual BER after application of physical layer error correcting techniques; the

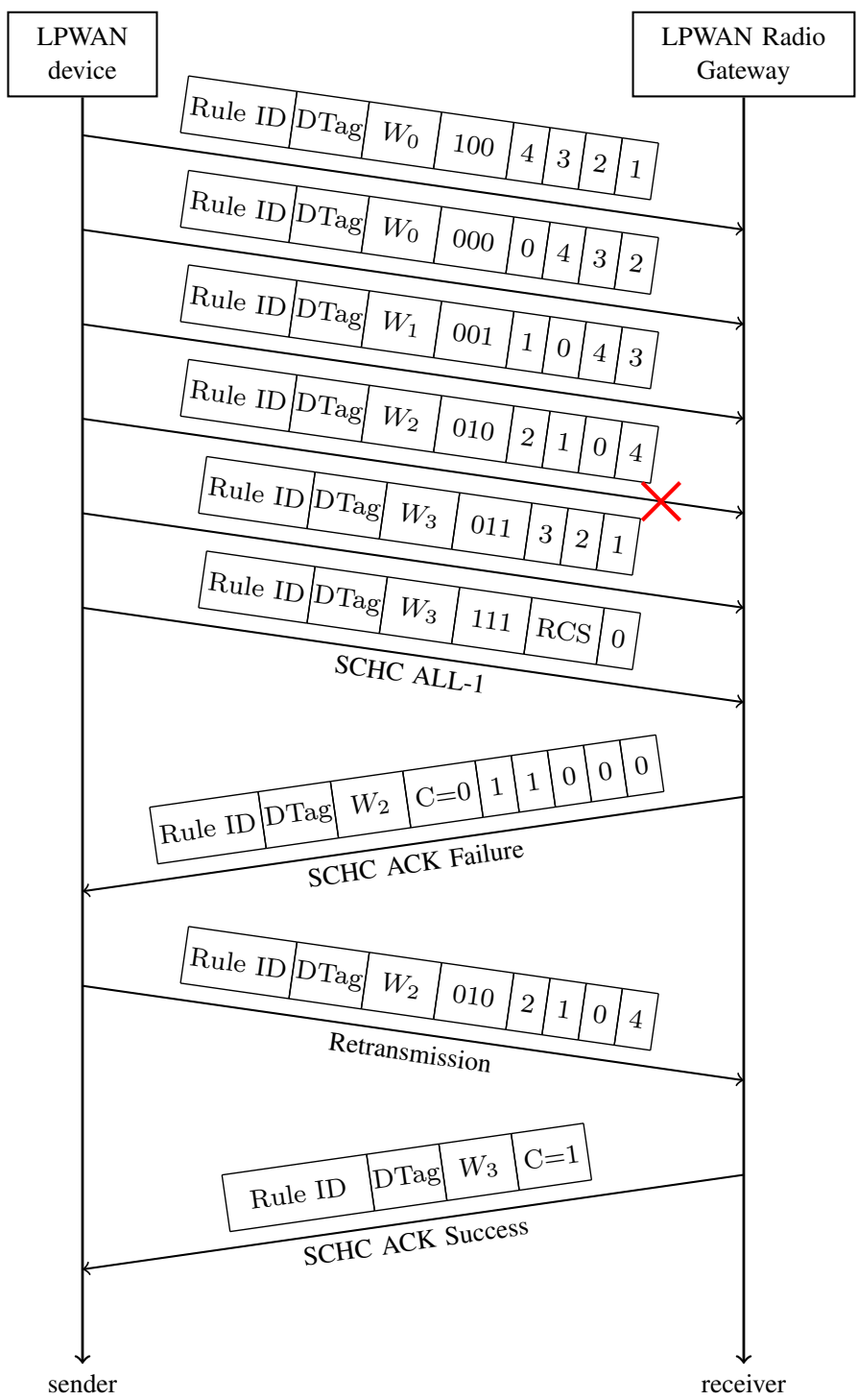

Fig. 7. Example of a transmission of a SCHC Packet with one lost SCHC Fragment in ACK-on-Error mode. Once the All-1 SCHC Message is received by the receiver, a SCHC ACK with $C=0$ and its corresponding bitmap is generated. The sender performs the SCHC Fragment retransmission. When the lost $\mathrm{SCHC}$ Fragment is received, the receiver performs the Integrity Check and sends the corresponding SCHC ACK Success indicating the end of the transmission of the SCHC Packet.

impact of the frame header size is considered separately, see Section V-C.) Assuming $P_{\mathrm{s}}$ fixed and all transmissions independent, the number of transmission attempts $N_{\mathrm{TA}}$ needed to successfully deliver a SCHC Fragment from the sender to the receiver follows a geometric distribution, i.e.,

$$
\mathbb{P}\left(N_{\mathrm{TA}}=n\right)=\left(1-P_{\mathrm{s}}\right)^{n-1} P_{\mathrm{s}} .
$$

Recall that at most only one ACK per window is generated: the ACK reports all the tiles not received in that window. Hence, until all SCHC Fragments containing all tiles of a window have been successfully received, a negative ACK is sent for that window and the missing SCHC Fragments are re-sent.

As a result, the number of such negative ACKs that are sent per window is the maximum number of retransmissions 
over all the SCHC Fragments in the window. The expected value of that number can be computed recursively, using a renewal argument. To do so, denote by $A_{j}$ the expected number of transmission attempt cycles (a cycle meaning that we send all the missing SCHC Fragments of a window once) until successful reception of all SCHC Fragments, when $j>$ 0 SCHC Fragments remain to be sent. Then, consider the situation after a transmission attempt cycle of all those $j$ SCHC Fragments: with probability $\left(\begin{array}{l}j \\ i\end{array}\right) P_{\mathrm{s}}^{j-i}\left(1-P_{\mathrm{s}}\right)^{i}$, there have been $j-i$ SCHC Fragments successfully received, and $i$ SCHC Fragments that need retransmission. From that situation, the expected number of transmission attempt cycles is simply $A_{i}$, hence the recursive relation

$$
A_{j}=1+\sum_{i=0}^{j}\left(\begin{array}{l}
j \\
i
\end{array}\right) P_{\mathrm{s}}^{j-i}\left(1-P_{\mathrm{s}}\right)^{i} A_{i},
$$

where the first term accounts for the transmission attempt we considered. Rearranging, we get

$$
A_{j}=\frac{1}{1+\left(1-P_{\mathrm{s}}\right)^{j}}\left[1+\sum_{i=0}^{j-1}\left(\begin{array}{c}
j \\
i
\end{array}\right) P_{\mathrm{s}}^{j-i}\left(1-P_{\mathrm{s}}\right)^{i} A_{i}\right]
$$

Using (4) with $A_{0}=0$, the number of ACKs required per window is simply $A_{k}-1$, removing the last successful transmission attempt cycle, when all the SCHC Fragments with all the tiles of that window were correctly received, with $k$ the number of SCHC Fragments in a window.

A SCHC Fragment can contain tiles from several consecutive windows, hence the number of SCHC Fragments to successfully send a window may vary. For a fixed window size $w$ (in tiles) and fragment size $f$ (in tiles), the number of SCHC Fragments per window $(k)$ can be modeled as a random variable, as follows:

$$
k= \begin{cases}k_{1}=\left\lfloor\frac{w}{f}\right\rfloor, & \text { with probability } 1+\left\lfloor\frac{w}{f}\right\rfloor-\frac{w}{f} \\ k_{2}=\left\lceil\frac{w}{f}\right\rceil, & \text { with probability } \frac{w}{f}-\left\lfloor\frac{w}{f}\right\rfloor,\end{cases}
$$

as illustrated in Fig. 8.

To compute $E_{k}$, the expected number of transmission attempts for $k_{1}$ and $k_{2}$ are first obtained from (4), and $E_{k}$ is the weighted average of $A_{k_{1}}-1$ and $A_{k_{2}}-1$ with the weights of (5). This gives us the $E_{k}$ for a given window size, fragment size and tile size, as follows:

$$
E_{k}=\left(1+\left\lfloor\frac{w}{f}\right\rfloor-\frac{w}{f}\right) \cdot A_{\left\lfloor\frac{w}{f}\right\rfloor}-1+\left(\frac{w}{f}-\left\lfloor\frac{w}{f}\right\rfloor\right) \cdot A_{\left\lceil\frac{w}{f}\right\rceil}-1 .
$$

\section{B. ACK Bit Overhead}

The ACK bit overhead $\left(O_{\mathrm{ACK}}\right)$ is defined as the average number of (negative) ACK bits sent for each data bit sent and provides the trade-off between the amount of data that can be transferred in a window and the resulting $\operatorname{ACK}(\mathrm{s})$ volume.

$O_{\mathrm{ACK}}$ is obtained by dividing $E_{k}$ by the window size and then multiplying the result by the ACK size $\left(L_{\mathrm{ACK}}\right)$. The window size can be obtained as the tile size $(t)$ multiplied by the number of tiles in a window $(w) . L_{\mathrm{ACK}}$ is equal to the bitmap size, that exactly equals $w$ (one bit per tile in a window) plus the ACK header $L_{\mathrm{SH}}$ (in bytes) allowing to express the ACK bit overhead as:

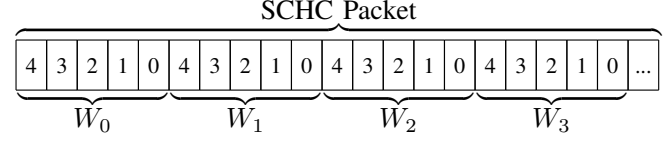

SCHC Fragments

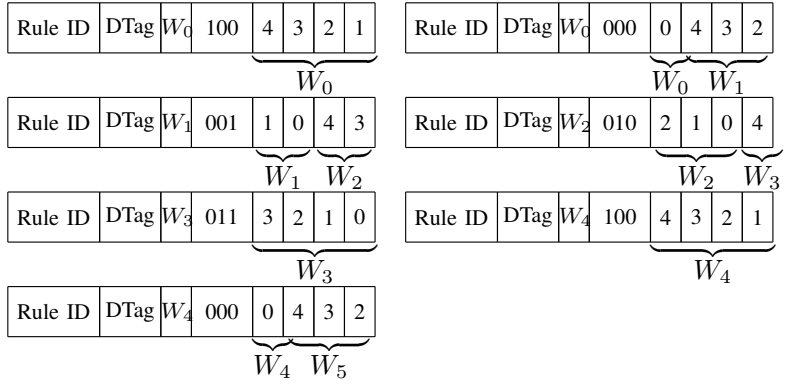

Fig. 8. Example of a SCHC Packet and SCHC Fragments. The SCHC Packet is fragmented in 5 tiles per window $(\boldsymbol{w}=5)$ and the SCHC Fragments can transport 4 tiles per fragment $(f=4)$. For a successful transmission of the packet, $\boldsymbol{W}_{0}$ needs $\boldsymbol{k}_{2}=\mathbf{2}$ successful SCHC Fragment transmissions, then $W_{1}, W_{2}$, and $W_{3}$ need each $k_{1}=1$ more successful SCHC Fragment transmission, in compliance with (5): a proportion $\frac{w}{f}-\left\lfloor\frac{w}{f}\right\rfloor=1 / 4$ of windows need $k_{2}=\left\lceil\frac{w}{f}\right\rceil$ new SCHC Fragment transmissions, whereas the other windows need only $k_{1}=\left\lfloor\frac{w}{f}\right\rfloor=1$ new SCHC Fragment transmission.

$$
O_{\mathrm{ACK}}=\frac{\left(8 L_{\mathrm{SH}}+w\right) \cdot E_{k}}{8 w t} .
$$

$O_{\mathrm{ACK}}$ quantifies the relation between the quantity of data and the quantity of ACKs that need to be sent. The ACK size $\left(L_{\mathrm{ACK}}\right)$ is related to the tile size. For the same window size (in bytes), larger tiles produce smaller bitmaps, thus smaller ACKs. Recall that the $O_{\mathrm{ACK}}$ is from the point of view of the SCHC framework layer, as it only considers the SCHC Headers.

\section{ACK Bit Overhead with L2 Headers}

As the SCHC framework sits on top of a LPWAN technology, there is an extra overhead due to L2 headers. To consider the additional cost involved in sending an ACK, the L2 header (with size $L_{\mathrm{L} 2 \mathrm{H}}$ in bytes) is added to $O_{\mathrm{ACK}}$ in the downlink, i.e. a penalty for sending an ACK, and can be calculated as follows:

$$
O_{\mathrm{ACK}_{\mathrm{L} 2}}=\frac{\left(8 \cdot\left(L_{\mathrm{L} 2 \mathrm{H}}+L_{\mathrm{SH}}\right)+w\right) \cdot E_{k}}{8 w t} .
$$

The ACK bit overhead with $\mathrm{L} 2$ headers $\left(O_{\mathrm{ACK}}{ }_{\mathrm{L} 2}\right)$ analyzes the impact of the $\mathrm{L} 2$ overhead and its relation with the window and tile sizes. Minimizing $O_{\mathrm{ACK}}$ maximizes the uplink data while optimizing the ACK size and volume, reducing the utilization of the LPWAN gateway and leveraging the available resources in duty-cycle-constrained networks.

\section{Percentage of used bits per fragment}

The percentage of used bits per SCHC Fragment provides information on how efficient a tile size is for a given L2 MTU $(F)$ considering the SCHC Headers. Due to LPWAN technologies capacity constraints, the tile size must be set to maximize the SCHC Fragment payload. 
Therefore, the percentage of usage of a SCHC Fragment, which we will denote by $P_{U}$, equals

$$
P_{U}=\frac{f \cdot t}{F-L_{\mathrm{SH}}} \cdot 100 .
$$

In LPWAN technologies such as LoRaWAN, $F$ can change during an on-going fragmented packet transmission [10] and the tile size previously chosen may not be multiple of the modified $F$ as it was in the previous one, leaving some bytes unused. The number of unused bytes in a SCHC Fragment $\left(f_{\text {unused }}\right)$, for a given tile size, is calculated as follows:

$$
f_{\text {unused }}=\left(F-L_{\mathrm{SH}}\right)-(f \cdot t) .
$$

For example, a 9-byte tile $(t=9)$ will use all the bytes when $F=11, f=1$ with $L_{\mathrm{SH}}=2$, but when having 5 tiles $(f=5)$ of 9 bytes and $F=53,6$ bytes per SCHC Fragment will not be used.

\section{E. Maximum window and bitmap sizes}

The SCHC framework defines a method for $\mathrm{F} / \mathrm{R}$ on the uplink to transfer SCHC Packets, but it does not propose a SCHC ACK F/R method. Without a fragmentation method the ACK is limited to one L2 MTU. Therefore, there is a maximum bitmap size (MBS) that leads to a maximum window size (MWS). Moreover, the tile size will limit the maximum amount of data on a window. The maximum bitmap size in tiles, i.e., number of bits in the bitmap, is therefore calculated as follows:

$$
\mathrm{MBS}=8 \cdot\left(F-L_{\mathrm{SH}}\right),
$$

and the maximum window size in bytes is then:

$$
\operatorname{MWS}=(t \cdot \mathrm{MBS})-U .
$$

\section{Performance Evaluation}

In this section, we use the models derived in section $\mathrm{V}$ to evaluate the ACK-on-Error mode in terms of the performance metrics presented. The section pursues two main goals. The first one is determining the impact of the main SCHC F/R ACK-on-Error mode parameters, i.e. window and tile sizes, and loss rate, on the considered performance metrics. The second goal is deriving the optimal settings for both window and tile sizes in a wide range of scenarios.

The fragment sizes $F$ used in the performance evaluation range between the minimum $(F=11)$ and maximum $(F=$ 242) MTU values in LoRaWAN for US $915 \mathrm{MHz}$ band (US915) and EU $868 \mathrm{MHz}$ band (EU868), respectively. These settings allow analyzing the performance of ACK-on-Error mode for the whole range of values for $F$ in LoRaWAN. Note that the physical layer configuration in LoRaWAN (including data unit size, and thus fragment size) is determined by the Data Rate (DR) and Spreading Factor (SF) [10]. The SCHC $\mathrm{ACK}$ and SCHC Fragment Header size used is 2 bytes $\left(L_{\mathrm{SH}}=\right.$ 2).

\section{A. ACK Message Overhead}

Fig. 9a shows the ACK message overhead $E_{k}$ as a function of $P_{\mathrm{s}}$, for different window sizes, and for $F=11$ and $t=9$ bytes so that $f=1$ (one tile per fragment), $k=w$, and there are no unused bits. As expected, $E_{k}$ decreases with $P_{\mathrm{s}}$. The difference in $E_{k}$ between a small window size (e.g., $w=1$ ) and a large window (e.g., $w=143$ ) is larger for lower $P_{\mathrm{s}}$ than with higher $P_{\mathrm{s}}$. This happens because $E_{k}$ has a logarithmic behavior as a function of $k$ (see Fig. 9b) and "flattens" when $P_{\mathrm{s}}$ increases: for small values of $k$ and $P_{\mathrm{s}}$, a small variation in $k$ produces large changes in $E_{k}$. The $E_{k}$ variation decreases as $k$ increases. For higher $P_{\mathrm{s}}$, the difference in $E_{k}$ is less dependent on $k$, since less ACKs are produced.

For $P_{\mathrm{s}}=1$, only one positive $\mathrm{ACK}$ is generated at the end of the fragmented packet transmission because no SCHC Fragments are lost, but $E_{k}=0$ because it only counts negative ACKs, i.e. failed window transmission cycles.

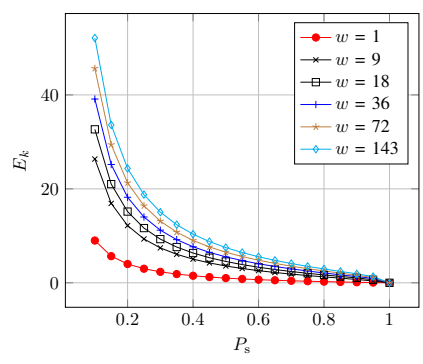

(a) $E_{k}$ vs $P_{\mathrm{s}}$

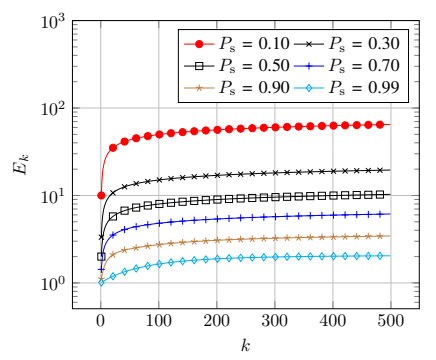

(b) $E_{k}$ vs $k$
Fig. 9. $\boldsymbol{E}_{\boldsymbol{k}}$ for $\boldsymbol{F}=\mathbf{1 1}, \boldsymbol{t}=\mathbf{9}$ and $\boldsymbol{L}_{\mathrm{SH}}=\mathbf{2}$ for different window sizes as a function of $\boldsymbol{P}_{\mathbf{S}}$ (a) and $\boldsymbol{k}(\mathrm{b})$.

\section{B. ACK Bit Overhead}

We now consider the ACK bit overhead metric $O_{\mathrm{ACK}}$ defined in (7), with the aim of minimizing that metric to obtain an optimal tile and window size.

1) Optimal Tile Size: Fig. 10a shows the impact of the tile size on $O_{\mathrm{ACK}}$, for $F=11$ and $P_{\mathrm{s}}=0.9$. For small tiles, $O_{\mathrm{ACK}}$ increases faster with the window size than for large tiles; indeed, a smaller tile size will require a larger bitmap for each data bit transferred, since the bitmap size equals the number of tiles in a window, hence is inversely proportional to the tile size for a fixed window size (in data volume). As the tile size increases, the ACK size required for the same window size decreases. When the window size increases, the ACK size becomes more relevant in $O_{\mathrm{ACK}}$ than the average number of ACKs, because the average number of ACKs has a logarithmic behavior (see Fig. 9b) while the ACK size increases linearly with the window size. The tile size that yields the smallest ACK size and the lowest $O_{\mathrm{ACK}}$ ratio is the largest possible tile size.

The results for $F=51$ and $F=242$ for $P_{\mathrm{s}}=0.9$ are shown in Fig. 10c and Fig. 10d, respectively. As in the previous case, a tile size equal to the SCHC Fragment payload size minimizes $O_{\mathrm{ACK}}$. As expected, there is a large difference between using a 9-byte and 49-byte tile, when compared to a 240-byte tile, as Fig. 10d shows. 


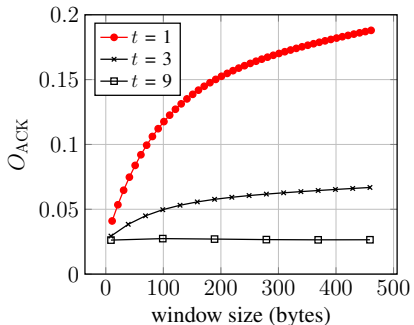

(a) $F=11, P_{\mathrm{s}}=0.9$

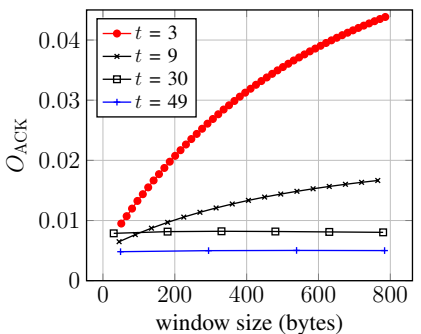

(c) $F=51, P_{\mathrm{s}}=0.9$

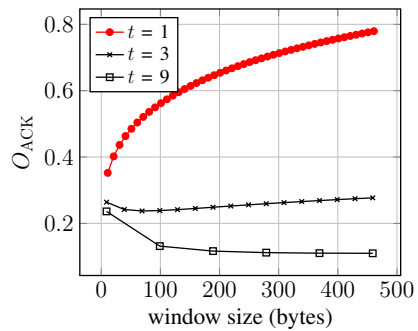

(b) $F=11, P_{\mathrm{S}}=0.5$

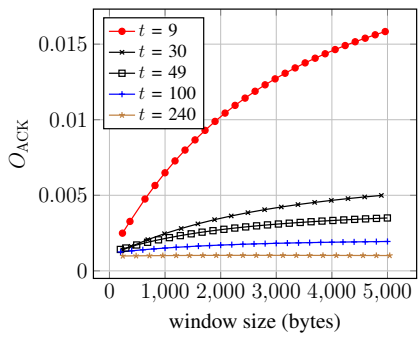

(d) $F=242, P_{\mathrm{S}}=0.9$
Fig. 10. $\boldsymbol{O}_{\mathrm{ACK}}$ vs window size. Impact of the tile size $\boldsymbol{t}$ on the $\boldsymbol{O}_{\mathrm{ACK}}$ $L_{\mathrm{SH}}=\mathbf{2}$.

Fig. 10b shows $O_{\mathrm{ACK}}$ for $P_{\mathrm{s}}=0.5$ and $F=11$, evaluated for different window sizes. For larger tile sizes, as the window size increases, $O_{\mathrm{ACK}}$ decreases because larger windows are more efficient when reporting many lost fragments.

For all the displayed settings, the optimal tile size is the largest possible tile, i.e., a tile size of the SCHC Fragment payload size, independently of $P_{\mathrm{s}}$. This occurs because the largest tile size maximizes the SCHC Fragment payload while reducing the number of bits in the bitmap, i.e. only one bitmap bit is required for each SCHC Fragment. Hence, as a practical recommendation, for technologies with fixed L2 MTU such as Sigfox, the optimal tile size is simply the SCHC Fragment payload size. For technologies with variable L2 MTU size, such as LoRaWAN, it is not possible to use a tile of the SCHC Fragment payload size in the larger fragment sizes because the tile has to fit in the smallest fragment size to support variable L2 MTU. In the case the SCHC Fragment size is known beforehand, the Rule can be chosen with the optimal tile and window sizes. If the L2 MTU changes, then the tile size can change accordingly.

2) Optimal Window Size: Now, the tile size is set to a fixed value, i.e., equal to the SCHC Fragment payload size. Fig. 11a and Fig. 11b illustrate the $O_{\mathrm{ACK}}$ as a function of $P_{\mathrm{s}}$ for different window sizes, for $F=11, t=9$ and $F=51$, $t=49$, respectively. When $P_{\mathrm{s}}$ is low, many fragments are lost and a larger window size leads to lower $O_{\mathrm{ACK}}$.

The interest of having large windows lies in what we can call ACK pooling, that is the fact that when several fragments (of the same window) fail, the information of the failures is pooled into a single ACK message. Large window sizes benefit from ACK pooling when $P_{\mathrm{s}}$ is low because one large ACK can report many lost fragments. Conversely, smaller windows sizes will generate a greater number of ACKs leading to higher overhead.

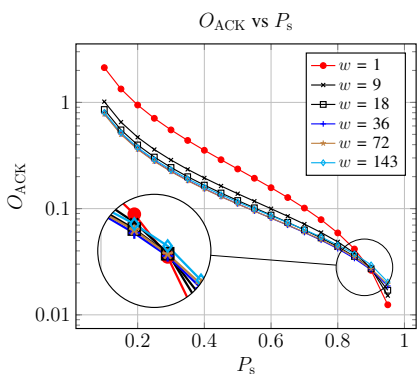

(a) $F=11, t=9$

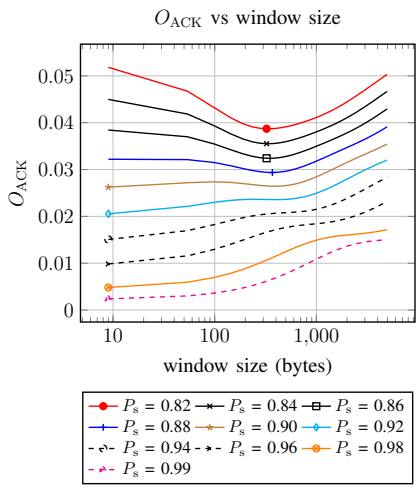

(c) $F=11, t=9$ (b) $F=51, t=49$

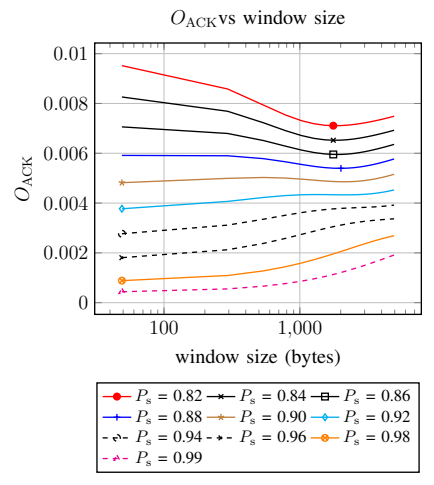

(d) $F=51, t=49$

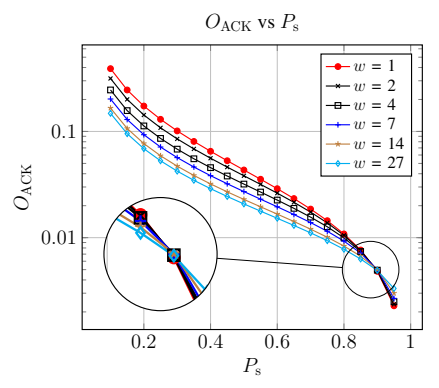

Fig. 11. Impact of the window size $\boldsymbol{w}$ (in tiles) or $\boldsymbol{w} \boldsymbol{t}$ (in bytes) on the ACK overhead $\boldsymbol{O}_{\text {ACK }}$. The optimal window size $\left(\boldsymbol{w}_{\text {opt }}\right)$ is indicated in (c) and (d) with a mark. $L_{\mathrm{SH}}=2$.

Henceforth, the tradeoff is between ACK pooling (larger windows mean less ACKs) and ACK size (larger windows mean larger ACKs), that we manage through the total ACK volume metric $O_{\mathrm{ACK}}$

As $P_{\mathrm{s}}$ increases, there exists one point (see zoom in Fig. 11a and Fig. 11b) beyond which smaller windows outperform larger window sizes. From this point forward, having smaller ACKs becomes more important since the number of losses is low. For example, the window size of 1 tile performs worst for lower $P_{\mathrm{S}}$ but as $P_{\mathrm{s}}$ increases, it becomes the one yielding the lowest $O_{\mathrm{ACK}}$. When $P_{\mathrm{s}}$ is greater than 0.9 , the window size of just 1 tile is optimal because rarely a SCHC Fragment will get lost, in which case a smaller ACK will be sent (almost no ACK pooling, and smaller ACKs). In contrast, large windows require a large ACK to report the few lost fragments.

As a consequence, there exists an optimal window size that minimizes $O_{\mathrm{ACK}}$, which depends not only on $P_{\mathrm{s}}$, but also on $F$ and $t$. Fig. 11c and Fig. 11d show the values for $O_{\mathrm{ACK}}$ for different windows sizes, and for $F=11, t=9$ and $F=51$, $t=49$, respectively. When $P_{\mathrm{s}}$ is 0.8 , ACK pooling benefits the larger windows and the optimal window size is large, as for example, 342 bytes $(w=38)$ in Fig. 11c. As $P_{\mathrm{s}}$ increases, $O_{\mathrm{ACK}}$ increases with the window size and for $P_{\mathrm{s}} \geq 0.9$ the benefit of ACK pooling is lost, leading to an optimal window size of one tile $(w=1)$.

Fig. 12a and Fig. 12b illustrate the results for $F=240$ with $t=49$ and $t=240$, respectively. When the tile size is small compared with the SCHC Fragment payload size $(f>1)$ there is no gain from ACK pooling for $P_{\mathrm{s}}>0.5$. 


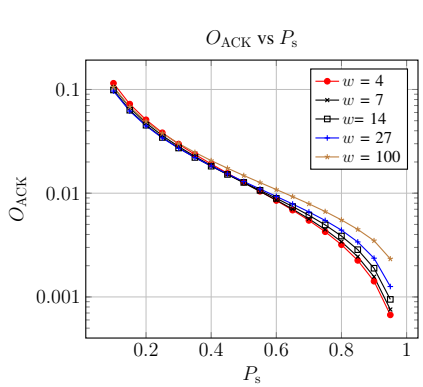

(a) $F=242, t=49$

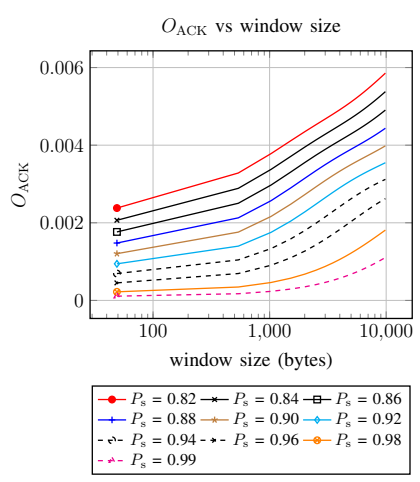

(c) $F=242, t=49$

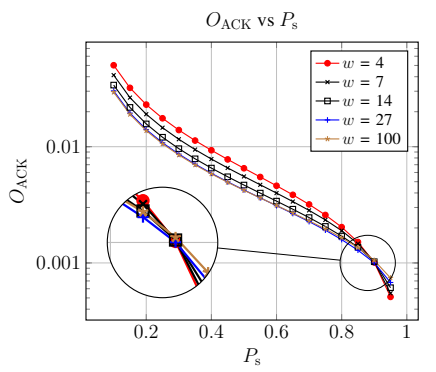

(b) $F=242, t=240$

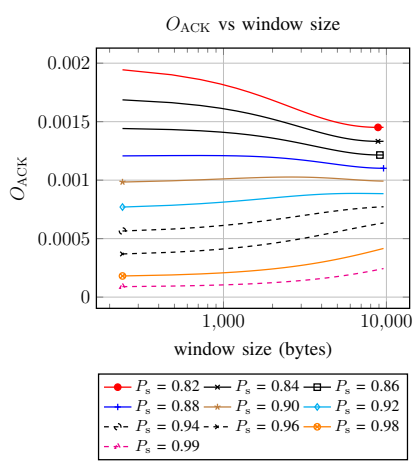

(d) $F=242, t=240$

Fig. 12. Impact of the window size $\boldsymbol{w}$ (in tiles) or $\boldsymbol{w} \boldsymbol{t}$ (in bytes) on the ACK overhead $\boldsymbol{O}_{\text {ACK }}$. The optimal window size $\left(\boldsymbol{w}_{\text {opt }}\right)$ is indicated in (c) and (d) with a mark. $\boldsymbol{L}_{\mathbf{S H}}=\mathbf{2}$.

Hence smaller windows perform better, as Fig. 12a shows. As the window size increases, more small tiles per SCHC Fragment are required when compared with larger tile sizes (see Fig. 12b), making the larger window size yield a greater $O_{\mathrm{ACK}}$ when using smaller tiles. Fig. 12c shows how $O_{\mathrm{ACK}}$ is minimum for small window sizes when $P_{\mathrm{s}}>0.80$ for $F=240$ and $t=49$ : the optimal window size is the smaller window, i.e. $w=4$. By contrast, Fig. 12d illustrates for $F=242$ and $t=240$ how a larger tile size will yield a larger optimal window size and benefit from ACK pooling for $P_{\mathrm{s}}<0.9$.

Hence, depending on the parameters and the channel conditions, the window size minimizing $O_{\mathrm{ACK}}$ varies. We now focus on that optimal window size.

Fig. 13a presents the optimal window size as a function of $P_{\mathrm{s}}$, for different $F$ values. When $P_{\mathrm{s}}$ is below 0.8 , in most of the cases, the optimal window size is large. The optimal window size decreases as $P_{\mathrm{s}}$ increases. As expected, the importance of ACK pooling decreases with respect to the ACK size. Fig. 13b presents the optimal window sizes for different $P_{\mathrm{s}}$ and for larger $F$ values. As for the case of smaller $F$, as $P_{\mathrm{s}}$ becomes higher, the optimal window size becomes smaller.

\section{ACK Bit Overhead with L2 Headers}

The previous two subsections (i.e. VI-A and VI-B) considered the ACK overhead as defined in (7), i.e., ignoring L2 headers in the size of ACKs. This may be justified if the operator charges the user based on the volume of L2 payloads. In this section we investigate the impact of counting the whole L2 ACK size by counting those headers, as suggested in (8).

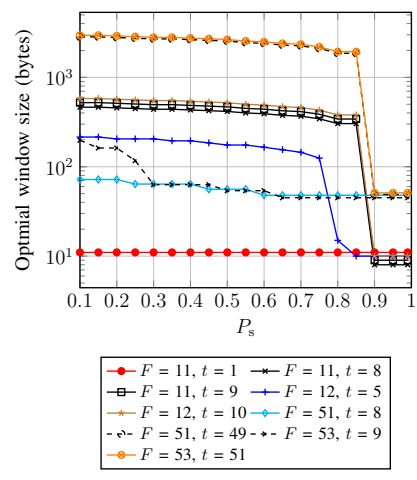

(a) $F=11,12,51,53$ (b) $F=115,125,242$

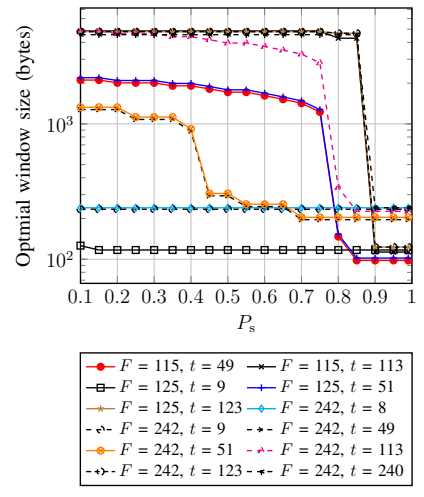

Fig. 13. Optimal window size vs $\boldsymbol{P}_{\mathbf{S}}$ for different settings.

The L2 header size of Sigfox according to [4] is 21 bytes in downlink with a payload of 8 bytes. The L2 headers of LoRaWAN according to [10] are 13 byte long, hence we will especially focus on those values.

For clarity in the figures, we define $P_{\mathrm{f}}$ as $1-P_{\mathrm{s}}$. Fig $14 \mathrm{a}$ and Fig. $14 \mathrm{~b}$ illustrate $O_{\mathrm{ACK}}$ for $F=11, t=9$ and for $F=242, t=49$, with $L_{\mathrm{L} 2 \mathrm{H}}=13$, respectively.

Smaller windows outperform larger ones for $P_{\mathrm{s}}>0.99$ (see the zoom in Figs. 14a and 14b), whereas in Section VI-B it is for $P_{\mathrm{s}}>0.90$. This happens because the additional constant length added to the ACK size favors the ACK pooling effect of large windows over the additional ACK length, making it more efficient to send one large ACK than several smaller ones (and their large L2 headers). Hence, only with very large success probability $P_{\mathrm{s}}>0.99$, i.e., $P_{\mathrm{f}}<10^{-2}$, does the ACK length effect take over the ACK pooling effect, as Fig. 14a shows. For $P_{\mathrm{s}}>0.99$, the best window size is 1 tile $(w=1)$, but as $P_{\mathrm{s}}$ decreases, the optimal window size increases (e.g., $w=143$ for $\left.P_{\mathrm{s}}<0.99\right)$.

Fig. 14c and Fig. 14d confirm our conclusions, by showing $O_{\mathrm{ACK}_{\mathrm{L} 2}}$ for $F=11, t=9$ and for $F=242, t=49$ with $L_{\mathrm{L} 2 \mathrm{H}}=13$ for different $P_{\mathrm{s}}$, respectively.

\section{Percentage of used bits per fragment}

The percentage of used bits per fragment $\left(P_{U}\right)$ provides an overview of how efficient a tile size is for a given SCHC Fragment size. Fig. 15a illustrates $P_{U}$ for $F=11,12,51,53$ and different tile sizes. When fragment payload sizes are a multiple of the tile size, $P_{U}$ is $100 \%$, e.g., $t=1 \forall F, t=3$ for $F=11, t=5$ for $F=12, t=7$ for $F=51$ and $t=17$ $F=53$. Furthermore, there are tile sizes that have a low $P_{U}$. For example, $t=5$ for $F=11$ only uses the $55.56 \%, t=6$ for $F=12$ with only $60 \%, t=25$ for $F=51$ with $51.02 \%$ and $t=26$ for $F=53$ with $50.98 \%$.

Fig. 15b shows the percentage of used bits for $F=$ 115, 125, 242. As with smaller SCHC Fragments, some tile sizes have a better $P_{U}$ than others. Moreover, as the tile size gets larger and only one tile can be fitted in the SCHC Fragment payload, the percentage of used bits is reduced significantly. For example, $t=57$ for $F=115$ with $50.44 \%$, 


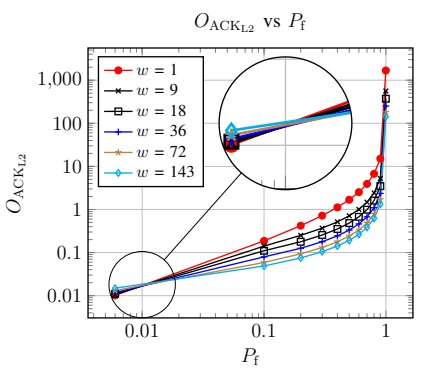

(a) $F=11, t=9$

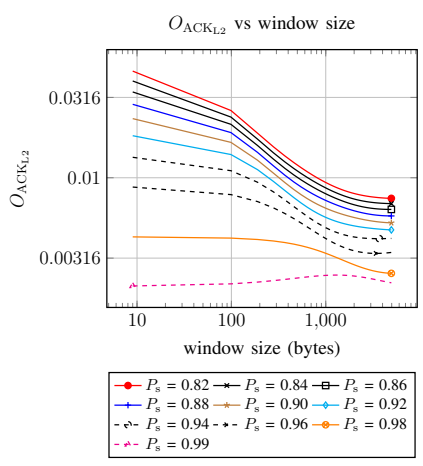

(c) $F=11, t=9$

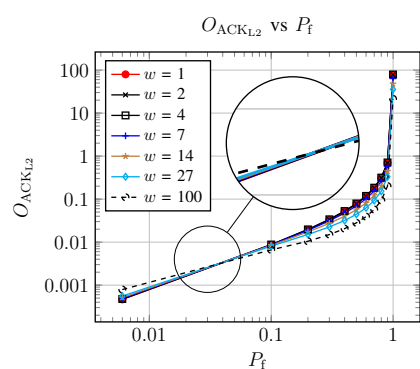

(b) $F=242, t=49$

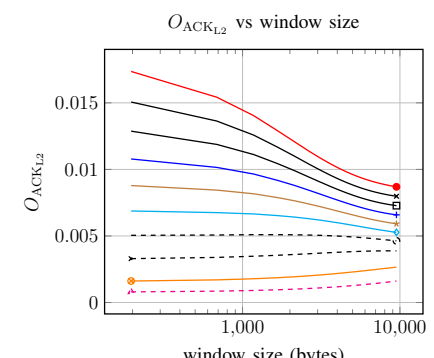

window size (bytes)

$\rightarrow P_{\mathrm{s}}=0.82 \rightarrow P_{\mathrm{s}}=0.84 \multimap P_{\mathrm{s}}=0.86$ $\begin{aligned} & \longrightarrow P_{\mathrm{s}}=0.88 \rightarrow-P_{\mathrm{s}}=0.90 \rightarrow-P_{\mathrm{s}}=0.92 \\ &-\leftrightarrow-P_{\mathrm{s}}=0.94 \rightarrow-P_{\mathrm{s}}=0.96 \rightarrow-P_{\mathrm{s}}=0.98\end{aligned}$ $\rightarrow P_{\mathrm{s}}=0.99$

(d) $F=242, t=49$
Fig. 14. Impact of the window size $\boldsymbol{w}$ (in tiles) or $\boldsymbol{w} \boldsymbol{t}$ (in bytes) on the ACK overhead $\boldsymbol{O}_{\mathrm{ACK}}$. The optimal window size $\left(\boldsymbol{w}_{\mathrm{opt}}\right)$ is indicated in (c) and (d) with a mark. $L_{\mathrm{SH}}=2$ and $L_{\mathrm{L} 2 \mathrm{H}}=13$.

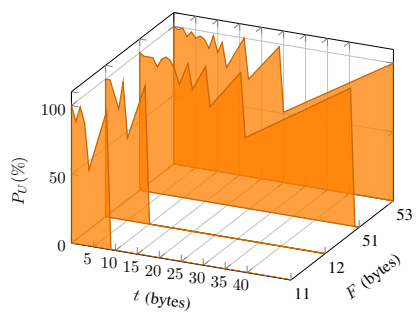

(a) $F=11,12,51,53$

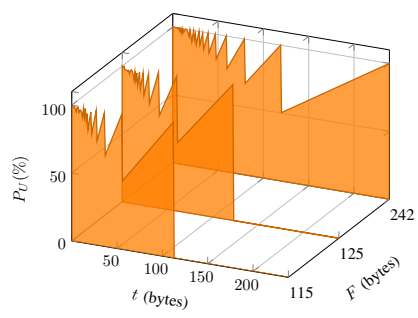

(b) $F=115,125,242$
Fig. 15. Percentage of usage $\boldsymbol{P}_{U}$ vs $t . \boldsymbol{L}_{\mathrm{SH}}=\mathbf{2}$.

$t=62$ for $F=125$ with $50.41 \%$ and $t=121$ for $F=242$ with $50.42 \%$.

\section{TEChNOLOgY-ORIEnTEd EVAluation}

The SCHC framework specification does not offer recommendations about how the ACK-on-Error parameter values should be selected. This section provides configuration guidance for, and discusses the impact of, the main parameters of ACK-on-Error mode when used over LoRaWAN (EU868 and US915) and Sigfox.

This section is organized as follows: section VII-A describes the physical layer parameters of LoRaWAN and Sigfox relevant to the ACK-on-Error mode configuration. Section VII-B provides our recommended values for the main ACK-on-Error parameters, along with the methodology used to determine such values. Section VII-C discusses the optimal window size results obtained. Finally, Section VII-D evaluates the global impact of limiting the ratio of LPWAN devices that use the optimal ACK-on-Error settings derived.

\section{A. LoRaWAN and Sigfox Relevant Parameters}

LoRaWAN defines a number of physical layer options for use in different world regions. In each region, a physical layer option corresponds to different settings of a parameter called the Spreading Factor (SF). Each SF defines a specific Data Rate (DR). In LoRaWAN, the L2 MTU depends on the SF/DR in use. All SF/DR settings in LoRaWAN are shown in the leftmost columns of Tables I and II, for the EU868 and US915 bands, respectively.

In contrast, Sigfox physical layer options are fixed, with only one constant L2 MTU (see Table III).

\section{B. Determining SCHC ACK-on-Error Mode Parameter Settings}

This section provides our recommended values for the main parameter settings for ACK-on-Error mode, and explains how they are obtained, for each considered technology, and as a function of Ps. Tables I to III and Fig. 16 present the ACKon-Error mode optimal parameter settings for LoRaWAN in EU868 and US915 bands, and for Sigfox, respectively. The main parameters comprise the fragment size (section VII-B.1), the tile size (section VII-B.2), the window size (section VIIB.3), the FCN field size $(N)$ (section VII-B.4), and the length of the Window field $(M)$ (section VII-B.5). Furthermore, reference values such as the maximum window size and the maximum bitmap size are also provided (section VII-B.6).

1) Fragment Size $(F)$ : In order to maximize efficiency, the value of $F$ needs to be equal to the current L2 MTU of the underlying LPWAN technology. Tables I to III show the optimal values of $F$ for LoRaWAN (Tables I and II), and for Sigfox (Table III). For LoRaWAN, the value of column $F$ depends on the current SF/DR settings (Tables I and II). For Sigfox, $F$ is a constant value (Table III).

2) Optimal Tile Size: Column $t$ of Tables I to III shows the most relevant tile sizes for each value of $F$ over LoRaWAN and Sigfox. For each value of $F$, the greatest tile sizes in column $t$ are the optimal sizes obtained in section VI-B. Recall that the optimal tile size is the one that entirely fills the SCHC Fragment payload. On the other hand, because in LoRaWAN the value of $F$ is variable, and SCHC F/R will still be possible when $F$ changes, the optimal tile sizes for all possible smaller values of $F$ are also evaluated. For example, the 49-byte tile size is optimal for $F=51$, and it is also evaluated for $F=115$ and $F=242$, for LoRaWAN EU868.

3) Optimal Window Size: The optimal window size $\left(w_{\text {opt }}\right)$ presented in Fig. 16 and in column $w_{\mathrm{opt}}$ of Table III is obtained by evaluating (8) from the minimum window size that generates an ACK size without padding (i.e. $w=8$ ) to the maximum window size in tiles derived from (11) (i.e. maximum number of bits in the bitmap). Then, the window size that yields the minimum $O_{\mathrm{ACK}}$ is selected. Finally, as the ACK size has to be a byte multiple, the optimal window size value is the next window size that will generate a bitmap that will not require padding (i.e. the next byte multiple). 


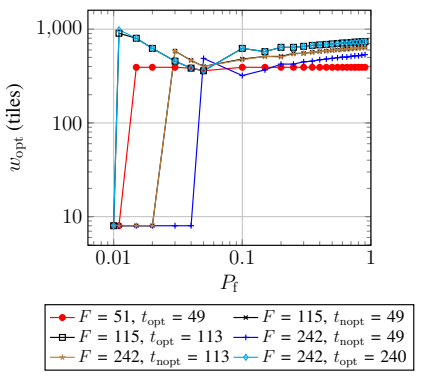

(a) $F$ for LoRaWAN EU868.

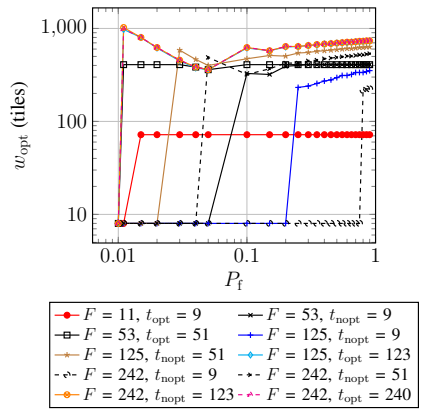

(b) $F$ for LoRaWAN US915.

Fig. 16. Optimal window size $\left(\boldsymbol{w}_{\text {opt }}\right)$ vs $\boldsymbol{P}_{\mathbf{f}}$.

Fig. 16 presents the values of $w_{\text {opt }}$ for LoRaWAN EU868 and LoRaWAN US915, evaluated for the corresponding values of $F$ and $t$ presented in Tables I and II, respectively. Table III presents the optimal window size for Sigfox. For the sake of clarity, Fig. 16 and subsequent figures are shown as a function of $P_{\mathrm{f}}$ (i.e. $1-P_{\mathrm{s}}$ ) instead of $P_{\mathrm{s}}$.

4) FCN Field Size $(N)$ : The value of $N$ is obtained as the minimum value that allows to unambiguously identify all tiles per window for a selected $w . N$ is obtained as:

$$
N=\left\lceil\log _{2}(w)\right\rceil .
$$

In Tables I and II, the value of column $N$ is determined for the optimal window size $\left(w_{\text {opt }}\right)$ presented in Figs. 16a and 16b, respectively. In Table III, the value of column $N$ is obtained for the values presented in the $w_{\text {opt }}$ column.

5) Window Field Length (M): The value of $M$ must be set to identify the number of windows required to carry a SCHC Packet size $\left(L_{S C H C}\right)$ and, it can be derived as:

$$
M= \begin{cases}1, & \text { if } L_{\mathrm{SCHC}} \leq w \cdot t \\ \left\lceil\log _{2}\left(\frac{L_{\mathrm{SCHC}}}{w \cdot t}\right)\right\rceil, & \text { if } L_{\mathrm{SCHC}}>w \cdot t .\end{cases}
$$

The values of columns $M$ in Tables I and II are obtained for the $w_{\mathrm{opt}}$ values presented in Fig. 16a and 16b, respectively. In Table III, the value of column $M$ is determined for the values shown in the $w_{\text {opt }}$ column. In Tables I to III, we considered two SCHC Packet sizes: 320 bytes and 1280 bytes. The first one is a relatively small size that will require fragmentation for all values of $F$ evaluated. The second one is a larger size that corresponds to the minimum packet size that needs to be supported by the layer below IPv6 [19].

6) Maximum Window and Bitmap Sizes: The SCHC framework does not define a fragmentation method for SCHC ACKs. Therefore, the L2 MTU of each technology limits the corresponding Maximum Bitmap Size (MBS) and Maximum Window Size (MWS) as explained in Section V-E. Table IV shows the MBS and MWS for LoRaWAN (EU868 and US912) and Sigfox with $L_{\mathrm{SH}}=2$, and $U=4$. Different tile sizes are presented considering different ACK-on-Error parameters configurations. Note that MBS does not depend on the tile size, but on the SCHC Fragment and SCHC Header sizes as shown in (11). MWS depends on MBS, the tile size and the RCS size $(U)$, as shown in (12). Sigfox has a downlink payload size of

TABLE I

ACK-ON-ERROR CONFIGURATION PARAMETERS FOR $\boldsymbol{w}_{\text {opt }}$ IN TABLE I

\begin{tabular}{|c|c|c|c|c|c|c|c|}
\hline \multirow{3}{*}{ SF } & \multirow{3}{*}{ DR } & \multirow{3}{*}{$\begin{array}{c}\mathbf{F} \\
\text { (bytes) }\end{array}$} & \multirow{3}{*}{$\begin{array}{c}t \\
\text { (bytes) }\end{array}$} & \multirow{3}{*}{$P_{\mathrm{s}}$} & \multirow{3}{*}{$\underset{\text { (bits) }}{\mathbf{N}}$} & \multicolumn{2}{|c|}{$\begin{array}{c}\text { M } \\
\text { (bits) }\end{array}$} \\
\hline & & & & & & SCHC & Packet size \\
\hline & & & & & & $\begin{array}{c}320 \\
\text { (bytes) }\end{array}$ & $\begin{array}{c}1280 \\
\text { (bytes) }\end{array}$ \\
\hline \multirow{2}{*}{$\begin{array}{l}12 \\
11 \\
10\end{array}$} & \multirow{2}{*}{$\begin{array}{l}\text { DR0, } \\
\text { DR1, } \\
\text { DR2 }\end{array}$} & \multirow{2}{*}{51} & \multirow{2}{*}{49} & $0<P_{\mathrm{s}} \leq 0.98$ & 8 & \multirow{2}{*}{1} & 1 \\
\hline & & & & $0.98<P_{\mathrm{s}} \leq 1$ & 3 & & 2 \\
\hline \multirow{8}{*}{9} & \multirow{8}{*}{ DR3 } & \multirow{8}{*}{115} & \multirow{4}{*}{49} & $0<P_{\mathrm{S}} \leq 0.80$ & 9 & \multirow{4}{*}{1} & \multirow{3}{*}{1} \\
\hline & & & & $0.80<P_{\mathrm{s}} \leq 0.90$ & 8 & & \\
\hline & & & & $0.90<P_{\mathrm{s}} \leq 0.97$ & 10 & & \\
\hline & & & & $0.97<P_{\mathrm{s}} \leq 1$ & 3 & & 2 \\
\hline & & & \multirow{4}{*}{113} & $0<P_{\mathrm{s}} \leq 0.95$ & 9 & \multirow{4}{*}{1} & \multirow{4}{*}{1} \\
\hline & & & & $0.95 \leq P_{\mathrm{S}}<0.98$ & 8 & & \\
\hline & & & & $0.98 \leq P_{\mathrm{S}}<0.99$ & 9 & & \\
\hline & & & & $0.99 \leq P_{\mathrm{S}}<1$ & 3 & & \\
\hline \multirow{11}{*}{$\begin{array}{l}8, \\
7\end{array}$} & \multirow{11}{*}{$\begin{array}{l}\text { DR4, } \\
\text { DR5 }\end{array}$} & \multirow{11}{*}{242} & \multirow{3}{*}{49} & $0<P_{\mathrm{S}} \leq 0.30$ & 9 & \multirow{3}{*}{1} & \multirow{2}{*}{1} \\
\hline & & & & $0.30<P_{\mathrm{s}} \leq 0.95$ & 8 & & \\
\hline & & & & $0.95<P_{\mathrm{S}} \leq 1$ & 3 & & 2 \\
\hline & & & & $0<P_{\mathrm{S}} \leq 0.80$ & 9 & \multirow{4}{*}{1} & \multirow{4}{*}{1} \\
\hline & & & \multirow{3}{*}{113} & $0.80<P_{\mathrm{S}} \leq 0.96$ & 8 & & \\
\hline & & & & $0.96 \leq P_{\mathrm{S}}<0.97$ & 9 & & \\
\hline & & & & $0.97 \leq P_{\mathrm{s}}<1$ & 3 & & \\
\hline & & & & $0 \leq P_{\mathrm{s}} \leq 0.90$ & 9 & & \\
\hline & & & 240 & $0.90<P_{\mathrm{S}}<0.97$ & 8 & 1 & 1 \\
\hline & & & 240 & $0.97 \leq P_{\mathrm{s}}<0.98$ & 9 & & \\
\hline & & & & $0.99 \leq P_{\mathrm{S}}<1$ & 3 & & \\
\hline
\end{tabular}
FOR LORAWAN EU868

8 bytes [4], hence with $L_{\mathrm{SH}}=2$, at most 6 bytes can be used for the bitmap.

\section{Optimal Window Size Results: Discussion}

In this subsection, we discuss the obtained optimal window size results for LoRaWAN and Sigfox, in terms of $P_{\mathrm{f}}$, shown in Fig. 16 and Table III, respectively.

As shown in Fig. 16, for high $P_{\mathrm{f}}$, the optimal window size is large, due to the benefit of ACK pooling. As $P_{\mathrm{f}}$ decreases, ACK pooling becomes less advantageous. Therefore, it is better to configure smaller window sizes, which leads to smaller ACK sizes.

Another observation from Fig. 16 is that the optimal window size fluctuates for larger values of $F$, as function of $P_{\mathrm{f}}$. In general, the $O_{\mathrm{ACK}}$ curve as a function of $P_{\mathrm{f}}$ has a "U" shape (see Figs. 11, 12 and 14). This happens because for small $w$, a great number of small ACKs are generated, whereas for large $w$, few large ACKs are generated. Therefore, a minimum value of $O_{\mathrm{ACK}} \mathrm{L} 2$ can generally be found between the two ends. However, the value of $P_{\mathrm{f}}$ affects the "U" shape. For very low $P_{\mathrm{f}}$, there are very few ACKs. In such a case, small $w$ produces short ACKs, producing a decay of the left side of the "U" shape of the curve as $P_{\mathrm{f}}$ decreases. Moreover, as $P_{\mathrm{f}}$ decreases, the value of $O_{\mathrm{ACK}}$ will tend to decrease. On the other hand, for large $w, E_{k}$ grows slowly with $w$ (see Fig. 9b). The combination of all these effects produces changes in the "U" shape in such a way that small changes in $P_{\mathrm{f}}$ generate fluctuations in $w_{\text {opt }}$ as a function of $P_{\mathrm{f}}$. This behavior can 
TABLE II

ACK-ON-ERror Configuration Parameters for $\boldsymbol{w}_{\text {opt }}$ IN TABLE II FOR LORAWAN US915

\begin{tabular}{|c|c|c|c|c|c|c|c|}
\hline \multirow{3}{*}{ SF } & \multirow{3}{*}{ DR } & \multirow{3}{*}{$\begin{array}{c}\mathbf{F} \\
\text { (bytes) }\end{array}$} & \multirow{3}{*}{$\begin{array}{c}t \\
\text { (bytes) }\end{array}$} & \multirow{3}{*}{$P_{\mathrm{s}}$} & \multirow{3}{*}{$\underset{\text { (bits) }}{\mathbf{N}}$} & \multicolumn{2}{|c|}{$\begin{array}{c}\text { M } \\
\text { (bits) }\end{array}$} \\
\hline & & & & & & SCHC & acket size \\
\hline & & & & & & $\begin{array}{c}320 \\
\text { (bytes) }\end{array}$ & $\begin{array}{c}1280 \\
\text { (bytes) }\end{array}$ \\
\hline \multirow{2}{*}{10} & \multirow{2}{*}{ DR0 } & \multirow{2}{*}{11} & \multirow{2}{*}{9} & $0<P_{\mathrm{S}}<0.98$ & 6 & 1 & 2 \\
\hline & & & & $0.98 \leq P_{\mathrm{S}} \leq 1$ & 4 & 3 & 5 \\
\hline \multirow{3}{*}{9} & \multirow{3}{*}{ DR1 } & \multirow{3}{*}{53} & 9 & $0<P_{\mathrm{S}} \leq 1$ & 8 & 1 & 1 \\
\hline & & & \multirow{2}{*}{51} & $0<P_{\mathrm{S}} \leq 0.98$ & 8 & \multirow{2}{*}{1} & 1 \\
\hline & & & & $0.98<\bar{P}_{\mathrm{s}} \leq 1$ & 3 & & 3 \\
\hline \multirow{11}{*}{8} & \multirow{11}{*}{ DR2 } & \multirow{11}{*}{125} & \multirow{3}{*}{9} & $0<P_{\mathrm{S}} \leq 0.60$ & 8 & \multirow{2}{*}{1} & \multirow{2}{*}{1} \\
\hline & & & & $0.60<P_{\mathrm{S}} \leq 0.80$ & 7 & & \\
\hline & & & & $0.80<P_{\mathrm{S}} \leq 1$ & 4 & 3 & 4 \\
\hline & & & \multirow{4}{*}{51} & $0<P_{\mathrm{S}} \leq 0.80$ & 9 & \multirow{4}{*}{1} & \multirow{3}{*}{1} \\
\hline & & & & $0.80<P_{\mathrm{S}} \leq 0.96$ & 8 & & \\
\hline & & & & $0.96<P_{\mathrm{S}} \leq 0.97$ & 9 & & \\
\hline & & & & $0.97<P_{\mathrm{S}} \leq 1$ & 3 & & 2 \\
\hline & & & \multirow{4}{*}{123} & $0<P_{\mathrm{S}} \leq 0.90$ & 9 & \multirow{4}{*}{1} & \multirow{4}{*}{1} \\
\hline & & & & $0.90<P_{\mathrm{S}} \leq 0.97$ & 8 & & \\
\hline & & & & $0.97<P_{\mathrm{S}} \leq 0.98$ & 9 & & \\
\hline & & & & $0.98<P_{\mathrm{S}} \leq 1$ & 3 & & \\
\hline \multirow{14}{*}{$\begin{array}{l}7, \\
8\end{array}$} & & & & $0<P_{\mathrm{S}} \leq 0.50$ & 7 & & 1 \\
\hline & & & 9 & $0.50<P_{\mathrm{S}} \leq 0.60$ & 5 & 1 & 2 \\
\hline & & & & $0.60<P_{\mathrm{S}} \leq 1$ & 5 & & 3 \\
\hline & & & & $0<P_{\mathrm{S}}<0.30$ & 9 & & 1 \\
\hline & & & 51 & $0.30 \leq P_{\mathrm{S}} \leq 0.95$ & 8 & 1 & 1 \\
\hline & & & & $0.95 \leq P_{\mathrm{s}} \leq 1$ & 3 & & 2 \\
\hline & DR3, & 24 & & $0<P_{\mathrm{S}} \leq 0.90$ & 9 & & \\
\hline & DR4 & & 123 & $0.90<P_{\mathrm{s}} \leq 0.97$ & 8 & 1 & 1 \\
\hline & & & & $0.97<P_{\mathrm{s}} \leq 0.98$ & 9 & & \\
\hline & & & & $0.98<P_{\mathrm{S}} \leq 1$ & 3 & & \\
\hline & & & & $0 \leq P_{\mathrm{S}} \leq 0.90$ & 9 & & \\
\hline & & & 240 & $0.90<P_{\mathrm{S}}<0.97$ & 8 & 1 & 1 \\
\hline & & & 240 & $0.97 \leq P_{\mathrm{S}}<0.98$ & 9 & 1 & 1 \\
\hline & & & & $0.99 \leq P_{\mathrm{s}}<1$ & 3 & & \\
\hline
\end{tabular}

be seen in Figs. 11 and 12 (except in Fig. 12c, where the considered tile size is non-optimal).

As shown in Fig. 16 and Table III, this fluctuation is neither present for LoRaWAN ( $F=11, F=51$ ) nor for Sigfox $(F=$ 12). The reason is that the MBS (see Table IV) is not large enough to allow $w_{\text {opt }}$ fluctuations to happen in the practical range given for those technology settings.

Note that $w_{\text {opt }}$ fluctuation increases with the L2 header size $\left(L_{\mathrm{L} 2 \mathrm{H}}\right)$. While in Fig. 13 (where $L_{\mathrm{L} 2 \mathrm{H}}=0$ ) there is little to no fluctuation of the optimal window size, in Fig. 16 the fluctuation is more pronounced. Indeed, the L2 header size contributes to the $O_{\mathrm{ACK}}$ metric as shown in (8).

As a practical remark, since the optimal window size depends on $P_{\mathrm{f}}$, an enhanced implementation of SCHC can estimate $P_{\mathrm{f}}$ and select the optimal window size accordingly. When using SCHC over LoRaWAN, the current value of $F$ needs to also be taken into account. Finally, note that the ACKon-Error parameter optimizations proposed in this paper do not require modifications to off-the-shelf radio platforms.
TABLE III

ACK-ON-ERROR CONFIGURATION PARAMETERS FOR $\boldsymbol{w}_{\text {opt }}$ FOR SIGFOX

\begin{tabular}{|c|c|c|c|c|c|c|}
\hline \multirow{2}{*}{$\begin{array}{c}\mathrm{F} \\
\text { (bytes) }\end{array}$} & \multirow{2}{*}{$\begin{array}{c}t \\
\text { (bytes) }\end{array}$} & \multirow{2}{*}{$P_{\mathrm{s}}$} & \multirow{2}{*}{$\begin{array}{l}w_{\text {opt }} \\
\text { (tiles) }\end{array}$} & \multirow{2}{*}{$\underset{\text { (bits) }}{\mathbf{N}}$} & \multicolumn{2}{|c|}{$\begin{array}{c}\text { M } \\
\text { (bits) }\end{array}$} \\
\hline & & & & & $\begin{array}{c}\text { SCHC } \\
320 \\
\text { (bytes) }\end{array}$ & $\begin{array}{c}\text { Packet Size } \\
1280 \\
\text { (bytes) }\end{array}$ \\
\hline \multirow{2}{*}{12} & \multirow{2}{*}{10} & $0 \leq P_{\mathrm{S}} \leq 0.95$ & 48 & 6 & 1 & 3 \\
\hline & & $0.95<P_{\mathrm{S}} \leq 1$ & 8 & 4 & 3 & 5 \\
\hline
\end{tabular}

$L_{\mathrm{SH}}=2$

TABLE IV

MAXIMUM BITMAP AND WINDOW SIZES FOR LORAWAN AND SIGFOX

\begin{tabular}{|c|c|c|c|c|c|}
\hline $\begin{array}{c}\text { Region \& } \\
\text { Technology }\end{array}$ & $\begin{array}{c}F \\
\text { (bytes) }\end{array}$ & $\begin{array}{c}t \\
\text { (bytes) }\end{array}$ & $\begin{array}{l}\text { MBS } \\
\text { (bits) }\end{array}$ & $\begin{array}{c}\text { MWS } \\
\text { (bytes) }\end{array}$ & $\underset{\text { (bits) }}{\mathbf{N}}$ \\
\hline \multirow{6}{*}{$\begin{array}{c}\text { LoRaWAN } \\
\text { EU868 }\end{array}$} & 51 & 49 & 392 & 19204 & 9 \\
\hline & \multirow{2}{*}{115} & 49 & \multirow{2}{*}{904} & 44292 & \multirow{2}{*}{10} \\
\hline & & 113 & & 102148 & \\
\hline & \multirow{3}{*}{242} & 49 & \multirow{3}{*}{1920} & 94076 & \multirow{3}{*}{11} \\
\hline & & 113 & & 216956 & \\
\hline & & 240 & & 460796 & \\
\hline \multirow{10}{*}{$\begin{array}{l}\text { LoRaWAN } \\
\text { US915 }\end{array}$} & 11 & 9 & 72 & 644 & 7 \\
\hline & \multirow{2}{*}{53} & 9 & \multirow{2}{*}{408} & 3668 & \multirow{2}{*}{9} \\
\hline & & 51 & & 20804 & \\
\hline & \multirow{3}{*}{125} & 9 & \multirow{3}{*}{984} & 8852 & \multirow{3}{*}{10} \\
\hline & & 51 & & 50180 & \\
\hline & & 123 & & 121028 & \\
\hline & \multirow{4}{*}{242} & 9 & \multirow{4}{*}{1920} & 17276 & \multirow{4}{*}{11} \\
\hline & & 51 & & 97916 & \\
\hline & & 123 & & 236156 & \\
\hline & & 240 & & 460796 & \\
\hline Sigfox & 12 & 10 & 48 & 476 & 6 \\
\hline
\end{tabular}

\section{Impact of Optimal ACK-on-Error Parameter Settings on Downlink Traffic}

We next illustrate the impact of the derived optimal ACKon-Error parameters values (shown in Tables I to III and Fig. 16) on the ACK-on-Error ACK traffic. To this end, we assume a network where LPWAN devices use ACK-on-Error mode to transmit SCHC Packets of the same size in the uplink, thus ACKs are sent in the downlink. We assume that only a fraction, $R_{\mathrm{opt}}$, of these devices are optimally configured. We define and evaluate two performance metrics: the ACK Excess Factor (AEF) and the ACK Bytes Excess Factor (BEF) for a given SCHC Packet size $\left(L_{\mathrm{SCHC}}\right)$. The AEF is the number of ACKs actually transmitted (for $R_{\mathrm{opt}} \leq 1$ ) divided by the number of ACKs sent when all devices are optimally configured $\left(R_{\mathrm{opt}}=1\right)$. Analogously, the BEF is the number of ACK bytes actually transmitted divided by the number of ACK bytes sent when $R_{\mathrm{opt}}=1$. Both metrics are evaluated considering the optimal window size $\left(w_{\text {opt }}\right)$ and a non-optimal window size $\left(w_{\text {nopt }}\right)$, for different values of $P_{\mathrm{f}}$. Further, a comparison between an optimal tile size $\left(t_{\mathrm{opt}}\right)$ and a nonoptimal tile size $\left(t_{\text {nopt }}\right)$ is performed for the same $w_{\text {opt }}$.

Note that the global impact of the proposed optimized 


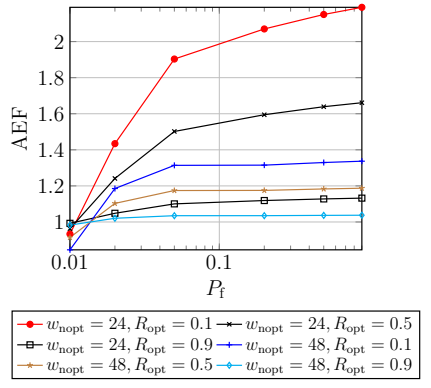

(a) AEF vs $P_{\mathrm{f}}$

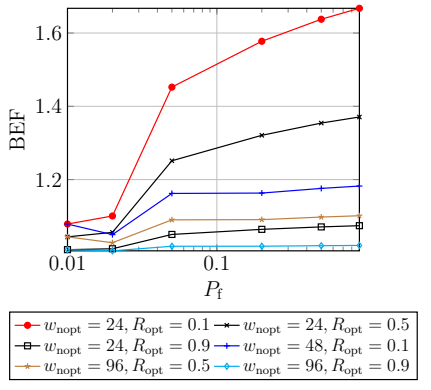

(b) BEF vs $P_{\mathrm{f}}$
Fig. 17. Impact of $\boldsymbol{w}_{\text {nopt }}$ on the AEF (a) and on the BEF (b) for different $\boldsymbol{R}_{\text {opt }}$ of devices using $\boldsymbol{w}_{\text {opt }}$. Results for LoRaWAN US915, $\mathrm{SF} 10 / \mathrm{DR} 0, \boldsymbol{F}=\mathbf{1 1}, \boldsymbol{L}_{\mathrm{SCHC}}=\mathbf{1 2 8 0}$, and $\boldsymbol{t}_{\mathrm{opt}}=\mathbf{9}$.

settings on the total downlink traffic in an LPWAN will depend also on other parameters that are independent of SCHC ACKon-Error mode. These include the number of devices that do not use SCHC, how often such devices request downlink messages, how many unsolicited downlink messages (e.g., management commands) are sent, etc.

Figs. $17 \mathrm{a}$ and $17 \mathrm{~b}$ show the $\mathrm{AEF}$ and $\mathrm{BEF}$ obtained for LoRaWAN US 915, SF10/DR0 ( $F=11$, see Table II), respectively, for $L_{\mathrm{SCHC}}=1280$ and different $R_{\mathrm{opt}}$ values, as a function of $P_{\mathrm{f}}$. The optimal window values are retrieved from Fig. 16b, that is, for $P_{\mathrm{f}} \leq 0.011, w_{\mathrm{opt}}=8$, and for $P_{\mathrm{f}}>0.011, w_{\text {opt }}=72$. The non-optimal window sizes ( $\left.w_{\text {nopt }}\right)$ evaluated are 24 and 48 tiles. The tile size used is the optimal one $\left(t_{\mathrm{opt}}=9\right)$.

As shown in Fig. 17, both AEF and BEF increases with $P_{\mathrm{f}}$. If all nodes use optimal settings, the number of ACKs and the amount of ACK bytes decrease by up to factors greater than 2 and 1.6, respectively, compared to the scenario where $R_{\mathrm{opt}}=0.1$ and $w_{\text {nopt }}=24$. As $R_{\text {opt }}$ increases, the potential for improvement decreases (e.g. for $R_{\mathrm{opt}}=0.9$ and $w_{\text {nopt }}=24$, the highest $\mathrm{AEF}$ and $\mathrm{BEF}$ values are 1.1 and 1.07, respectively). Note that ACK traffic improvement decreases as $w_{\text {nopt }}$ approaches the $w_{\text {opt }}$ value.

Another remarkable result shown in Fig. 17 is that, for $P_{\mathrm{f}} \leq$ 0.01 , the number of ACKs sent when optimal settings are used increases, as the AEF is lower than 1. However, there is a reduction in the excess of ACK bytes sent (i.e. BEF is greater than 1), leading to benefits in channel occupancy. In this range of $P_{\mathrm{f}}$ values, all window sizes considered generate a similar number of ACKs for the same SCHC Packet size, but $w_{\text {opt }}$ benefits from using the smallest ACK size.

Figs. 18a and $18 \mathrm{~b}$ show the $\mathrm{AEF}$ and $\mathrm{BEF}$ derived for LoRaWAN US915, SF10/DR0 $(F=11), L_{\mathrm{SCHC}}=1280$ when using $w_{\text {opt }}$ with an optimal tile size $\left(t_{\text {opt }}=9\right)$, compared to using a non-optimal tile size ( $\left.t_{\text {nopt }}\right)$, as a function of $P_{\mathrm{f}}$. Both the number of ACKs and the amount of ACK bytes may decrease by a factor of up to 2.7, depending on $R_{\text {opt }}$ and $t_{\text {nopt }}$. As $R_{\text {opt }}$ increases, and as $t_{\text {nopt }}$ approaches $t_{\text {opt }}$, the potential performance improvement decreases, since more devices use a $t$ configuration closer to the optimal one.

In general, when too many fragments are lost, it is better to send large ACKs, since in that case an ACK can be

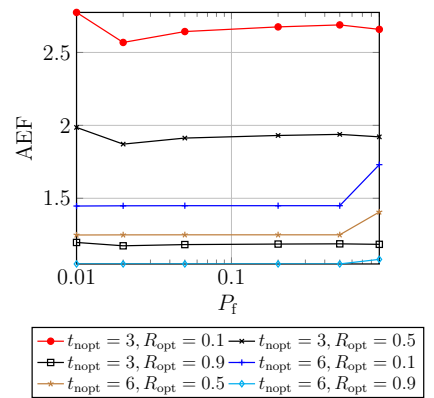

(a) AEF vs $P_{f}$

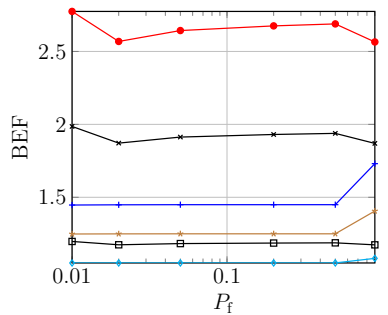

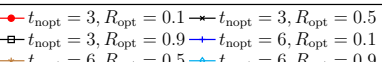

(b) $\mathrm{BEF}$ vs $P_{\mathrm{f}}$
Fig. 18. Impact of $\boldsymbol{t}_{\text {nopt }}$ on the AEF (a) and on the BEF (b) for different $\boldsymbol{R}_{\text {opt }}$ of devices using $\boldsymbol{t}_{\text {opt }}$. Results for LoRaWAN US915, $F=11, L_{\mathrm{SCHC}}=1280, N_{\mathrm{SCHC}}=100$, and $w_{\mathrm{opt}}$.

amortized to report many losses. Otherwise, using small ACKs is preferable. Recall that the ACK size is related to the number of tiles in a window. Assuming that fragmentation is performed in the uplink, the downlink channel usage by SCHC ACKon-Error can be optimized. This means that ACK traffic per data traffic can be minimized. This translates into lower billing and longer LPWAN device battery lifetime. From the LPWAN gateway perspective, it reduces its load, which is specially critical in duty-cycle restricted networks, and considering that a single gateway may serve a large number of LPWAN devices.

\section{CONCLUSIONS}

The SCHC framework enables LPWAN technologies to comply with IPv6 by performing a static context header compression and fragmentation. ACK-Always and ACK-onError modes, provide reliable F/R between the sender and the receiver. In this article, we have developed a mathematical model to analyze the ACK-on-Error mode, focusing on the number of ACKs required to transmit data. We were then able to perform an analysis that yielded to transmission parameters minimizing the ACK burden imposed on the (more sensitive to duty-cycle constraints) downlink. We then have applied our mathematical model to state-of-the-art LPWAN technologies such as LoRaWAN and Sigfox, to minimize the ACK bit overhead taking into account the retransmission process subtleties of the ACK-on-Error mode. Finally, we illustrate how the optimal parameter settings derived allow to decrease the ACK overhead.

\section{ACKNOWLEDGMENT}

This research was done during Sergio Aguilar visit at IMTAtlantique and is supported in part by the Spanish Government through project TEC2016-79988-P, AEI/FEDER, EU and by Secretaria d'Universitats i Recerca del Departament d'Empresa i Coneixement de la Generalitat de Catalunya 2017 SGR 376.

\section{REFERENCES}

[1] S. Farrell, "Low-Power Wide Area Network (LPWAN) Overview." RFC 8376, May 2018. 
[2] L. Casals, B. Mir, R. Vidal, and C. Gomez, "Modeling the energy performance of LoRaWAN," Sensors, vol. 17, no. 10, 2017.

[3] H. Mroue, A. Nasser, S. Hamrioui, B. Parrein, E. Motta-Cruz, and G. Rouyer, "MAC layer-based evaluation of IoT technologies: LoRa, SigFox and NB-IoT," in Proc. of IEEE MENACOMM, (Jounieh, Lebanon), April 2018.

[4] C. Gomez, J. C. Veras, R. Vidal, L. Casals, and J. Paradells, “A Sigfox Energy Consumption Model," Sensors, vol. 19, no. 3, 2019.

[5] A. Lavric, A. I. Petrariu, and V. Popa, "Long Range SigFox Communication Protocol Scalability Analysis Under Large-Scale, High-Density Conditions," IEEE Access, vol. 7, pp. 35816-35825, 2019.

[6] N. I. Osman and E. B. Abbas, "Simulation and Modelling of LoRa and Sigfox Low Power Wide Area Network Technologies," in Proc. of ICCCEEE, (Guayaquil, Ecuador), Aug 2018.

[7] A. Minaburo, L. Toutain, C. Gomez, D. Barthel, and J.-C. Zúñiga, "SCHC: Generic Framework for Static Context Header Compression and Fragmentation." RFC 8724, April 2020.

[8] C. Gomez, A. Minaburo, L. Toutain, D. Barthel, and J. C. Zuniga, "IPv6 over LPWANs: connecting Low Power Wide Area Networks to the Internet (of Things)," IEEE Wireless Communications, vol. 27, no. 1 pp. 206-213, 2020.

[9] F. Adelantado, X. Vilajosana, P. Tuset-Peiro, B. Martinez, J. MeliaSegui, and T. Watteyne, "Understanding the limits of LoRaWAN," IEEE Commun. Mag., vol. 55, pp. 34-40, Sept 2017.

[10] LoRa Alliance Technical Committee, "LoRaWAN 1.1 Specification," https://lora-alliance.org/sites/default/files/201804/lorawantm_specification_-v1.1.pdf, Oct 2017.

[11] Sigfox, "Sigfox connected objects: Radio specifications," https://build.sigfox.com/sigfox-device-radio-specifications, 22019. Rev. 1.3.

[12] K. Mekki, E. Bajic, F. Chaxel, and F. Meyer, "Overview of Cellular LPWAN Technologies for IoT Deployment: Sigfox, LoRaWAN, and NB-IoT," in Proc. of IEEE PerCom Workshops, (Athens, Greece), March 2018.

[13] I. Suciu, X. Vilajosana, and F. Adelantado, "An analysis of packet fragmentation impact in LPWAN," in Proc. of IEEE WCNC, (Barcelona, Spain), 2018.

[14] B. Moons, A. Karaagac, J. Haxhibeqiri, E. De Poorter, and J. Hoebeke, "Using SCHC for an optimized protocol stack in multimodal LPWAN solutions," in Proc. of IEEE WF-IoT, (Limerick, Ireland), 042019.

[15] W. Ayoub, F. Nouvel, S. Hmede, A. E. Samhat, M. Mroue, and J.-C. Prévotet, "Implementation of SCHC in NS-3 Simulator and Comparison with 6LoWPAN," in Proc. of 26th ICT, (Hanoi, Vietnam), Apr. 2019.

[16] K. Q. Abdelfadeel, V. Cionca, and D. Pesch, "LSCHC: Layered Static Context Header Compression for LPWANs," in Proc. of CHANTS, Session: Security \& IoT, (New York, USA), 2017.

[17] K. Q. Abdelfadeel, V. Cionca, and D. Pesch, "Dynamic Context for Static Context Header compression in LPWANs," in Proc. of 14th DCOSS, (New York, USA), June 2018.

[18] S. Aguilar, A. Marquet, L. Toutain, C. Gomez, R. Vidal, N. Montavont, and G. Z. Papadopoulos, "LoRaWAN SCHC Fragmentation Demystified," in Ad-Hoc, Mobile, and Wireless Networks (M. R. Palattella, S. Scanzio, and S. Coleri Ergen, eds.), pp. 213-227, Springer International Publishing (Cham), 2019.

[19] S. Deering and R. Hinden, "Internet Protocol, Version 6 (IPv6) Specification.” RFC 8200, July 2017. 